\title{
ON MAXIMAL TRANSITIVE SETS OF GENERIC DIFFEOMORPHISMS
}

\author{
by Christian BONATTI and Lorenzo J. DÍAZ*
}

\begin{abstract}
We construct locally generic $\mathrm{C}^{1}$-diffeomorphisms of 3-manifolds with maximal transitive Cantor sets without periodic points. The locally generic diffeomorphisms constructed also exhibit strongly pathological features generalizing the Newhouse phenomenon (coexistence of infinitely many sinks or sources). Two of these features are: coexistence of infinitely many nontrivial (hyperbolic and nonhyperbolic) attractors and repellors, and coexistence of infinitely many nontrivial (nonhyperbolic) homoclinic classes.

We prove that these phenomena are associated to the existence of a homoclinic class $\mathrm{H}(\mathrm{P}, f)$ with two specific properties:

- in a $\mathrm{C}^{1}$-robust way, the homoclinic class $\mathrm{H}(\mathrm{P}, f)$ does not admit any dominated splitting,

- there is a periodic point $\mathrm{P}^{\prime}$ homoclinically related to $\mathrm{P}$ such that the Jacobians of $\mathrm{P}^{\prime}$ and $\mathrm{P}$ are greater than and less than one, respectively.
\end{abstract}

\section{Introduction}

One of the main problems in dynamical systems is to describe the limit set for a large class of diffeomorphisms. The ideal objective is to split the limit set into finitely many pieces of dynamics which are mutually independent, maximal, and dynamically indecomposable. The next step should be to give a description (as complete as possible) of each piece.

In the case of hyperbolic diffeomorphisms, the Smale theory gives a complete topological description of the limit set: the limit set of a hyperbolic diffeomorphism is the union of finitely many pairwise disjoint hyperbolic transitive sets (existence of a dense orbit), these sets are the basic pieces of the Smale theory. These basic sets are locally maximal and correspond to homoclinic classes of periodic points, see [Sm]. These notions correspond to the ideas of dynamical independence, maximality, and indecomposability.

The Smale diffeomorphisms fail to be dense in the space of $\mathrm{C}^{1}$-diffeomorphisms if the dimension of the ambient manifold is strictly greater than two, see for instance the first examples in [AS] in dimension greater than or equal to 4, adapted for 3-manifolds in [Si], and its density remains an open problem for surface $\mathrm{C}^{1}$-diffeomorphisms. Thus, in the nonhyperbolic setting, it is natural to try to decompose the limit set of a large class of diffeomorphisms $f$ as the union of (preferably) finitely many elementary pieces of dynamics playing the role of the basic pieces of the Smale theory. For the role of elementary pieces of dynamics of

* This paper was partially supported by CNPq, Faperj, and Pronex Dynamical Systems (Brazil), PICS-CNRS and the Agreement Brazil-France in Mathematics. The authors acknowledge to IMPA and Laboratoire de Topologie, Université de Bourgogne, for the warm hospitality during their visits while preparing this paper. We also acknowledge M.-C. Arnaud, F. Béguin and the referees for their comments on the first version of this paper. 
a diffeomorphism $f$ defined on a compact manifold $\mathrm{M}$ there are three natural candidates:

- the robustly transitive sets introduced in [DPU]: an $f$-invariant set $\Lambda$ is robustly transitive if there is a neighborhood $U$ of it such that the set $\Lambda_{g}=\bigcap_{n \in \mathbf{Z}} g^{n}(\overline{\mathrm{U}})$ is transitive and contained in $\mathrm{U}$ for every $g \mathrm{C}^{1}$-close to $f$ and $\Lambda_{f}=\Lambda$.

A robustly transitive set $\Lambda$ is good for the role of elementary piece of dynamics if the neighborhood $\mathrm{U}$ in the definition can be chosen being a filtrating neighborhood, that is, $\mathrm{U}=\mathrm{V} \backslash \overline{\mathrm{W}}$, where $\mathrm{V}$ and $\mathrm{W}$ are open open sets such that $f(\overline{\mathrm{V}}) \subset \mathrm{V}$ and $f(\overline{\mathrm{W}}) \subset \mathrm{W}$. Otherwise, the robustly transitive set may be included in a greater transitive set.

- Given any periodic saddle $\mathrm{P}$ of $f$, the closure of the transverse intersections of the stable and unstable manifolds of the orbit of $\mathrm{P}$ is a transitive set, the homoclinic class of $\mathrm{P}$, denoted by $\mathrm{H}(\mathrm{P}, f)$.

- The maximal transitive sets, that is, sets which are maximal in the family of all transitive compact invariant sets of $f$ ordered by inclusion. As the closure of the union of an increasing (for $\subset$ ) family of transitive sets is transitive, Zorn's Lemma ensures that any transitive set is contained in a maximal one.

These three notions are closely related but, in general, they are not equivalent. We adopt here the generic or locally generic point of view: a property $\mathscr{P}$ is locally generic if there exist a non-empty open set $\mathscr{U}$ of $\operatorname{Diff}^{1}(\mathrm{M})$ and a residual subset $\mathscr{R}$ of $\mathscr{U}$ on which $\mathscr{P}$ is satisfied.

- By definition, every robustly transitive set associated to a filtrating neighbourhood is maximal transitive. Moreover, as a consequence of the Connecting Lemma in $[\mathrm{H}]$, robustly transitive sets of generic diffeomorphisms are relative homoclinic classes, where the relative homoclinic class of a saddle $\mathrm{P}$ in a neighbourhood $\mathrm{U}$ is the closure of the transverse homoclinic points of $\mathrm{P}$ whose orbits are contained in $\mathrm{U}$. However, in general, homoclinic classes (relative or not) fail to be robustly transitive sets (see, for instance, $\left.\left[\mathrm{BD}_{2}\right]\right)$.

- For generic $\mathrm{C}^{1}$-diffeomorphisms, every homoclinic class $\mathrm{H}(\mathrm{P}, f)$ is a maximal transitive set (see $[\mathrm{Ar}]$ ) verifying a stronger property: every transitive set intersecting $\mathrm{H}(\mathrm{P}, f)$ is contained in it (see $\left.[\mathrm{CMP}]^{1}\right)$.

Here we prove that generic diffeomorphisms may have maximal transitive sets which are neither homoclinic classes nor robustly transitive sets (this result

1 This stronger notion corresponds to the so-called maximal transitive sets in [CMP]. The name saturated transitive set we use here seems to be more coherent with the usual terminology, see Section 1.1. 
will be restated in a more precise and stronger formulation in the next section, see Theorem A):

Theorem. - Given any compact 3-manifold $\mathrm{M}$ there is a locally residual subset $\mathscr{F}(\mathrm{M})$ of Diff ${ }^{1}(\mathrm{M})$ of diffeomorphisms $f$ having maximal transitive Cantor sets $\Lambda_{f}$ without periodic points. In particular, the maximal transitive sets $\Lambda_{f}$ are not homoclinic classes.

The sets $\Lambda_{f}$ in the theorem are infinitely renormalizable according to the terminology in [BGLT] or adding machines, see for instance [BS].

Let us observe that, following [CMP], for generic diffeomorphisms, every maximal transitive set which is not a homoclinic class does not contain periodic points. So we define aperiodic maximal transitive sets to be the maximal transitive sets without periodic points. However, as a classical consequence of the Closing Lemma, see $[\mathrm{Pu}]$, every aperiodic maximal transitive set of a generic diffeomorphism is contained in the closure of the set of periodic points.

The construction of the aperiodic maximal transitive sets in the theorem involves the coexistence of infinitely many (different) homoclinic classes. Actually, due to a recent result in $[\mathrm{Ab}]$, the coexistence of infinitely many different (maybe trivial) homoclinic classes is a necessary condition for the (locally generic) existence of aperiodic maximal transitive sets. More precisely, [Ab] states that, in a residual set of $\operatorname{Diff}^{l}(\mathrm{M})$, the cardinality of the set of homoclinic classes is locally constant. Moreover, for those generic diffeomorphisms with finitely many homoclinic classes, there is a spectral decomposition theorem analogous to the one in the hyperbolic case (see [Sm]): the non-wandering set is the union of finitely many pairwise disjoint homoclinic classes exhibiting a weak form of hyperbolicity (existence of a dominated splitting, see Definition 3.7). Furthermore, as in the hyperbolic case, the homoclinic classes are the maximal invariant sets in neighborhoods corresponding to levels of a filtration. In particular, the maximal transitive sets of generic diffeomorphisms with finitely many homoclinic classes are homoclinic classes. This result in $[\mathrm{Ab}]$ generalizes the generic dichotomy (hyperbolicity versus infinitely many sinks or sources) obtained in [Ma] for generic $\mathrm{C}^{1}$-diffeomorphisms of surfaces.

Theorem A (locally generic existence of aperiodic maximal transitive sets) is consequence of a generalization of the so-called Newhouse Phenomenon (coexistence of infinitely many sinks or sources for locally generic diffeomorphisms). In the case of $\mathrm{C}^{2}$-diffeomorphisms, this phenomenon is associated to the unfolding of homoclinic tangencies, see $\left[\mathrm{N}_{1}\right],\left[\mathrm{N}_{2}\right]$ and $[\mathrm{PT}]$ for surface diffeomorphisms and $[\mathrm{PV}]$ and [Tj] in higher dimensions. In the $\mathrm{C}^{1}$-setting the existence of such a phenomenon for surface diffeomorphisms remains an open question.

In higher dimensions, the $\mathrm{C}^{1}$-Newhouse phenomenon (as well the locally generic existence of aperiodic maximal transitive sets) is related to the existence 
of wild homoclinic classes, see $\left[\mathrm{BD}_{2}\right]$. The homoclinic class $\mathrm{H}(\mathrm{P}, f)$ of a periodic point $\mathrm{P}$ is wild if (in a robust way) it does not admit any dominated splitting.

In dimension 3, a homoclinic class $\mathrm{H}(\mathrm{P}, f)$ is wild if it contains (in a stable way) a pair of hyperbolic periodic points of indices (dimension of the stable bundle) two and one whose derivatives have non-real (contracting and expanding, respectively) eigenvalues. We will write $\mathrm{H}(\mathrm{P}, f) \in \mathrm{W}_{0}(f)$ if furthermore there is a periodic point $\mathrm{P}^{\prime}$ homoclinically related to $\mathrm{P}$, such that the Jacobian of $f$ (at the period) at the points $\mathrm{P}$ and $\mathrm{P}^{\prime}$ is greater than 1 and less than 1 , respectively, see Definition 1.1.

Here we consider a non-empty open set $\mathscr{W}_{0}$ of diffeomorphisms $f$ (on a compact 3-manifold) having a wild homoclinic class $\mathrm{H}(\mathrm{P}, f) \in \mathrm{W}_{0}(f)$.

In the next section, in Theorem $\mathrm{B}$, we will give a much more precise statement of the following result:

Theorem. - Generic diffeomorphisms $f \in \mathscr{W}_{0}$ satisfy the following universal property: for every open set $\mathscr{O}$ of diffeomorphisms of the disk $\mathrm{D}^{3}$, there are infinitely many disjoint periodic disks on which the first return map of $f$ is smoothly conjugate to some element of $\mathscr{O}$.

As a consequence we get:

Corollary. - Generic diffeomorphisms $f \in \mathscr{W}_{0}$ display infinitely many times (in periodic disks with pairwise disjoint orbits) any robust property of $\mathrm{C}^{1}$-diffeomorphisms of the disk $\mathrm{D}^{3}$. In particular, these diffeomorphisms exhibit simultaneously:

- infinitely many pairwise disjoint non-trivial homoclinic classes,

- infinitely many non-trivial hyperbolic and non-hyperbolic attractors, and

- infinitely many non-trivial hyperbolic and non-hyperbolic repellors.

For a more complete list of pathological forms of the Newhouse Phenomenon see Corollary C.

In the proof of the previous theorem (see also Theorem B), the key technical result is that given any wild homoclinic class $\mathrm{H}(\mathrm{Q}, f) \in \mathrm{W}_{0}(f)$ of a diffeomorphism $f$ there are diffeomorphisms $g$ arbitrarily $\mathrm{C}^{1}$-close to $f$ with a periodic point $\mathrm{P}$ of arbitrarily large period $n$ and arbitrarily close to $\mathrm{H}(\mathrm{Q}, f)$ such that the derivative $\mathrm{D} g^{n}(\mathrm{P})$ is the identity, (see Theorem 3.2). This claim follows by using strongly the arguments in [BDP]. This assertion allows us to prove that given any diffeomorphism $f$ with a wild homoclinic class $\mathrm{H}(\mathrm{Q}, f) \in \mathrm{W}_{0}(f)$ and any open set $\mathscr{O}$ of diffeomorphisms of the disk $\mathrm{D}^{3}$ into itself preserving the orientation, there are disks $\mathrm{D}$ arbitrarily close to $\mathrm{H}(\mathrm{Q}, f)$ and a $\mathrm{C}^{1}$-perturbation $g$ of $f$ such that the disks $\mathrm{D}$ are periodic for $g$ (i.e., $\mathrm{D}, g(\mathrm{D}), \ldots, g^{m-1}(\mathrm{D})$ are pairwise disjoint and $g^{m}(\mathrm{D}) \subset \mathrm{D}$, for some $m \geq 1$ ) and the restriction of $g^{m}$ to $\mathrm{D}$ is differentially conjugate to some element of $\mathscr{O}$. 
Let us now explain how Theorem A follows from Theorem B. Consider any generic diffeomorphism $f$ with a wild homoclinic class in $\mathrm{W}_{0}(f)$. First, by definition, the property of having a homoclinic class in $\mathrm{W}_{0}(f)$ is an open one. So, Theorem B implies that there are small periodic disks where the first return map has a new wild homoclinic class. In other words, by rescaling a generic diffeomorphism $f$ with a wild homoclinic class in $\mathrm{W}_{0}(f)$, one obtains a new generic diffeomorphism $f_{1}$ with a wild homoclinic class in $\mathrm{W}_{0}\left(f_{1}\right)$. Arguing inductively, one gets an infinite decreasing sequence of small periodic disks $\mathrm{D}_{k}$ on which the first return maps of $f$ have wild homoclinic classes. Finally, the aperiodical maximal transitive sets of the theorem are obtained as the intersection of the orbits of the disks $\mathrm{D}_{k}$.

\subsection{Definitions and precise statement of results}

Before stating precisely our results let us give some definitions.

Let $\mathrm{M}$ be a compact closed manifold and $\operatorname{Diff}^{\mathrm{l}}(\mathrm{M})$ the space of $\mathrm{C}^{1}$-diffeomorphisms of $\mathrm{M}$ endowed with the usual $\mathrm{C}^{1}$-uniform topology. Consider $f \in$ $\operatorname{Diff}^{1}(\mathrm{M})$, we say that an $f$-invariant set $\Lambda$ is transitive if it is the closure of the forward orbit of some point $x$ of $\Lambda$. A transitive set $\Lambda$ is maximal if it is a maximal element of the family of all compact transitive sets of $f$ ordered by inclusion. A maximal transitive set is aperiodic if it does not contain any periodic orbit. A transitive set is saturated if every transitive set $\Sigma$ intersecting $\Lambda$ is contained in $\Lambda$ (this means that the transitive set is saturated by the equivalence relation generated by the relation of belonging to the same transitive set). Observe that, since the union of two transitive sets may fail to be transitive, it is not guaranteed that every transitive set is contained in a saturated one (recall that it is contained in a maximal one), see for instance the examples in [DS] of diffeomorphisms with a pair of different homoclinic classes with non-empty intersection whose union is not contained in any transitive set.

An $f$-invariant set $\Lambda$ of a diffeomorphism $f$ is minimal if the orbit of any point of $\Lambda$ is dense in itself (or equivalently, $\Lambda$ has no proper $f$-invariant sets). In particular, an infinite minimal set $\Lambda$ does not contain periodic orbits. An $f$ invariant set $\Lambda$ is uniquely ergodic if it supports only one $f$-invariant probability measure (which is necessarily ergodic). Observe that there are minimal sets where the restriction of $f$ is not uniquely ergodic (see $[\mathrm{Fu}]$ ).

The homoclinic class of a hyperbolic periodic point $\mathrm{P}$ of $f$, denoted by $\mathrm{H}(\mathrm{P}, f)$, is the closure of the transverse intersections of the orbits of the stable and unstable manifolds of $\mathrm{P}$. Every homoclinic class $\mathrm{H}(\mathrm{P}, f)$ is an $f$-invariant transitive set and the hyperbolic periodic points of the same index as $\mathrm{P}$ are dense in $\mathrm{H}(\mathrm{P}, f)$ (the index of a hyperbolic periodic point $\mathrm{Q}$ of $f$ is the dimension of the stable bundle 
of $f$ at $Q$, i.e., the number of eigenvalues of modulus less than one of $\mathrm{D} f^{p(\mathrm{Q})}(\mathrm{Q})$ counted with multiplicity, where $p(\mathbf{Q})$ is the period of $\mathbf{Q})$.

Two hyperbolic periodic points $\mathrm{P}_{1}$ and $\mathrm{P}_{2}$ of $f$ are homoclinically related if $\mathrm{W}^{s}\left(\mathrm{P}_{1}\right) \pitchfork \mathrm{W}^{u}\left(\mathrm{P}_{2}\right) \neq \varnothing$ and $\mathrm{W}^{u}\left(\mathrm{P}_{1}\right) \pitchfork \mathrm{W}^{s}\left(\mathrm{P}_{2}\right) \neq \varnothing$ (where $\mathrm{W}^{s}\left(\mathrm{P}_{1}\right) \pitchfork \mathrm{W}^{u}\left(\mathrm{P}_{2}\right)$ denotes the transverse intersection between $\mathrm{W}^{s}\left(\mathrm{P}_{1}\right)$ and $\left.\mathrm{W}^{u}\left(\mathrm{P}_{2}\right)\right)$. In particular, the indices of $\mathrm{P}_{1}$ and $\mathrm{P}_{2}$ are equal and their homoclinic classes coincide. In fact, the homoclinic class of a hyperbolic periodic point $\mathrm{P}$ is the closure of the periodic points homoclinically related to $\mathrm{P}$.

Finally, a compact set $\Lambda$ is Lyapunov stable for $f$ if for every neighborhood $\mathrm{U}$ of $\Lambda$ there is a neighborhood $\mathrm{V}$ of $\Lambda$ such that $f^{i}(\mathrm{~V}) \subset \mathrm{U}$ for all $i \geq 0$. Observe that every Lyapunov stable transitive set $\Lambda$ is saturated: any transitive set intersecting $\Lambda$ is contained in any neighborhood of $\Lambda$. This also holds if $\Lambda$ is Lyapunov stable for $f^{-1}$.

A set $\mathscr{F}$ of $\operatorname{Diff}^{1}(\mathrm{M})$ is residual in an open subset $\mathscr{U}$ of $\operatorname{Diff}^{1}(\mathrm{M})$ if there are open subsets $(\mathscr{V})_{n \in \mathbf{N}}$ of $\operatorname{Diff}^{1}(\mathrm{M})$ such that every $\mathscr{V}_{n}$ is dense in $\mathscr{U}$ and $\cap_{n} \mathscr{V}_{n} \subset \mathscr{F}$. We say that a set $\mathscr{F}$ is locally residual if there is a non-empty open subset $\mathscr{U}$ of $\operatorname{Diff}^{1}(\mathrm{M})$ in which $\mathscr{F}$ is residual.

Theorem A. - Given any closed 3-manifold $\mathrm{M}$ there is a locally residual subset $\mathscr{F}(\mathrm{M})$ of $\operatorname{Diff}^{1}(\mathrm{M})$ of diffeomorphisms $f$ having aperiodic maximal transitive Cantor sets $\Lambda_{f}$. Moreover, the set $\Lambda_{f}$ is minimal and uniquely ergodic for every $f \in \mathscr{F}(\mathrm{M})$. In particular, the maximal transitive sets $\Lambda_{f}$ are not homoclinic classes. Finally, the sets $\Lambda_{f}$ are simultaneously Lyapunov stable for $f$ and $f^{-1}$, hence the sets $\Lambda_{f}$ are saturated.

The constructions in the proof of this theorem also implies that every diffeomorphism $f \in \mathscr{F}(\mathrm{M})$ simultaneously has uncountable aperiodic maximal transitive sets. This remark was pointed out to us by F. Béguin.

Observe that given any hyperbolic periodic point $\mathrm{P}_{f}$ of $f$ there is a $\mathrm{C}^{1}$ neighborhood $\mathscr{V}$ of $f$ such that the continuation $\mathrm{P}_{g}$ of $\mathrm{P}_{f}$ is defined for every $g$ in $\mathscr{V}$.

Definition 1.1. - Consider a diffeomorphism $f$ defined on a closed three manifold $\mathrm{M}$ and a hyperbolic periodic point $\mathrm{P}_{f}$ of $f$.

1. The homoclinic class of $\mathrm{P}_{f}$ is wild if there is a neighborhood $\mathscr{V}_{0}$ of $f$ such that, for any $g \in \mathscr{V}_{0}$, the homoclinic class $\mathrm{H}\left(\mathrm{P}_{g}, g\right)$ does not admit any dominated splitting (see Definition 3.7 for the notion of dominated splitting).

2. The homoclinic class $\mathrm{H}\left(\mathrm{P}_{f}, f\right)$ belongs to $\mathrm{W}_{0}(f)$ if there are two hyperbolic periodic points $\mathrm{Q}_{f}$ and $\mathrm{P}_{f}^{\prime}$ and a neighborhood $\mathscr{V}$ of $f$ where the continuations $\mathrm{P}_{g}, \mathrm{P}_{g}^{\prime}$ and $\mathrm{Q}_{g}$ are defined for all $g \in \mathscr{V}$ such that

(a) $\mathrm{Q}_{g}$ belongs to $\mathrm{H}\left(\mathrm{P}_{g}, g\right)$,

(b) the point $\mathrm{P}_{g}$ has index two and a contracting non-real multiplier,

(c) the point $\mathbf{Q}_{g}$ has index one and an expanding non-real multiplier, 
(d) the point $\mathrm{P}_{g}^{\prime}$ has index two and is homoclinically related to $\mathrm{P}_{g}$,

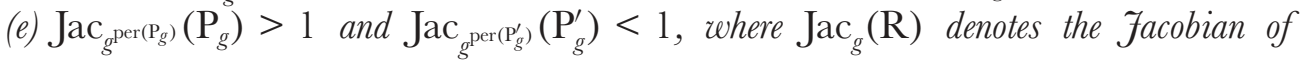
$g$ at the point $\mathrm{R}$.

We define homoclinic classes of points of index 1 in $\mathrm{W}_{0}(f)$ analogously.

Notice that items (a)-(c) of the definition of homoclinic classes in $\mathrm{W}_{0}(f)$ imply that the homoclinic class $\mathrm{H}\left(\mathrm{P}_{f}, f\right)$ is wild.

Denote by $\mathscr{W}(\mathrm{M})$ the set of diffeomorphisms of $\operatorname{Diff}^{-1}(\mathrm{M})$ having a wild homoclinic class, and by $\mathscr{W}_{0}(\mathrm{M})$ the set of diffeomorphisms $f$ having a homoclinic class $\mathrm{H}\left(\mathrm{P}_{f}, f\right) \in \mathrm{W}_{0}(f)$. By the previous comment, $\mathscr{W}_{0}(\mathrm{M}) \subset \mathscr{W}(\mathrm{M})$.

By definition, the sets $\mathscr{W}(\mathrm{M})$ and $\mathscr{W}_{0}(\mathrm{M})$ are open in $\operatorname{Diff}^{\mathrm{l}}(\mathrm{M})$. The fact that the set $\mathscr{W}_{0}(\mathrm{M})$ is non-empty can be proved exactly as in $\left[\mathrm{BD}_{2}\right.$, Section 3.2], where it is proved that the set $\mathscr{W}(\mathrm{M})$ is non-empty, see the appendix (Section 6) in this paper for details.

The arguments in $\left[\mathrm{BD}_{2}\right]$ show that $\mathrm{C}^{1}$-generic diffeomorphisms of $\mathscr{W}_{0}(\mathrm{M})$ exhibit simultaneously infinitely many sinks and infinitely many sources. In fact, in [BDP] it is proved that, for generic diffeomorphisms, any homoclinic class either has a dominated splitting or is contained in the closure of the (infinite) set of sinks and sources. Thus generic diffeomorphisms of $\mathscr{W}(\mathrm{M})$ (i.e., with a wild homoclinic class) have infinitely many sinks or sources.

Remark 1.2. - The proof of Theorem A shows that diffeomorphisms with aperiodic maximal transitive sets are generic in the set $\mathscr{W}_{0}(\mathrm{M})$.

We prove that generic diffeomorphisms of $\mathscr{W}_{0}(\mathrm{M})$ are the universal models for 3-dimensional dynamics, in the sense that they satisfy the following universal property.

Let $\mathrm{D}^{n}$ be the compact ball of radius 1 in $\mathbf{R}^{n}$ and denote by $\operatorname{Diff}_{\text {int }}^{+}\left(\mathrm{D}^{n}\right)$ the space of orientation preserving $\mathrm{C}^{1}$-diffeomorphisms $\phi: \mathrm{D}^{n} \rightarrow \operatorname{int}\left(\mathrm{D}^{n}\right)$ endowed with the usual $\mathrm{C}^{1}$-topology.

Definition 1.3 (Universal Dynamics). - A diffeomorphism $f$ has universal dynamics at an $f$-invariant set $\Sigma$ if for every open subset $\mathscr{O}$ of $\operatorname{Diff}_{\text {int }}^{+}\left(\mathrm{D}^{n}\right)$ and every point $x \in \Sigma$ there are sequences of pairwise disjoint disks $\left(\mathrm{D}_{k}\right)_{k}$ and of natural numbers $\left(n_{k}\right)_{k}$ and a constant $\mathrm{K}>0$ such that:

(U1) the disks $\mathrm{D}_{k}, f\left(\mathrm{D}_{k}\right), \ldots, f^{n_{k}-1}\left(\mathrm{D}_{k}\right)$ are pairwise disjoint,

(U2) the disks $\mathrm{D}_{k}$ satisfy the following:

- $\operatorname{diam}\left(\mathrm{D}_{k}\right) \rightarrow 0$ and $\mathrm{D}_{k} \rightarrow x$ as $k \rightarrow \infty$,

- $\left(\sup \left\{\operatorname{diam}\left(f^{i}\left(\mathrm{D}_{2 k}\right)\right)\right\}_{i=0}^{n_{2 k}-1}\right) \rightarrow 0$ as $k \rightarrow \infty$, and

- $\left(\sup \left\{\operatorname{diam}\left(f^{-i}\left(\mathrm{D}_{2 k+1}\right)\right)\right\}_{i=0}^{n_{2 k+1}-1}\right) \rightarrow 0$ as $k \rightarrow \infty$, 
(U3) $f^{n_{2 k}}\left(\mathrm{D}_{2 k}\right) \subset \operatorname{int}\left(\mathrm{D}_{2 k}\right)$ and the restriction $\left.f^{n_{2 k}}\right|_{\mathrm{D}_{2 k}}$ of $f^{n_{2 k}}$ to $\mathrm{D}_{2 k}$ is differentially conjugate to some $g_{2 k} \in \mathscr{O}$,

(U4) $f^{-n_{2 k+1}}\left(\mathrm{D}_{2 k+1}\right) \subset \operatorname{int}\left(\mathrm{D}_{2 k+1}\right)$ and the restriction $\left.f^{-n_{2 k+1}}\right|_{\mathrm{D}_{2 k+1}}$ is differentially conjugate to some $g_{2 k+1} \in \mathscr{O}$.

The following result is the key of all the constructions in this paper.

Theorem B. - There is a residual subset $\mathscr{U}(\mathbf{M})$ of $\mathscr{W}_{0}(\mathrm{M})$ of diffeomorphisms $f$ with universal dynamics at any wild homoclinic class in $\mathrm{W}_{0}(f)$.

Theorem A will follow from Theorem B. We shall see that for every $f$ in the residual subset $\mathscr{U}(\mathrm{M})$ of $\mathscr{W}_{0}(\mathrm{M})$ there are natural numbers $n_{k}$ and disks $\mathrm{D}_{k}$ as in the definition of Universal Dynamics where the induced dynamics of $f$ (i.e., the restriction of $f^{n_{k}}$ or $f^{-n_{k}}$ to $\mathrm{D}_{k}$, according to the case) belongs to $\mathscr{W}_{0}\left(\mathrm{D}_{k}\right)$ (the set of wild diffeomorphisms $g$ of $\mathrm{D}_{k}$ having a homoclinic class in $\mathrm{W}_{0}(g)$ ). This fact allows us to apply Theorem $\mathrm{B}$ to this small disk $\mathrm{D}_{k}$. Arguing inductively and repeating the previous construction infinitely many times, we will get maximal transitive sets $\Phi(f)$ which are infinitely renormalizable: there are sequences of nested disks $\left(\Delta_{k}\right)_{k}$ and of natural numbers $\left(n_{k}\right)$ satisfying conditions (U1)-(U4) above such that

$$
\Phi(f)=\bigcap_{2 k} \hat{\Delta}_{2 k}, \quad \text { where } \quad \hat{\Delta}_{2 k}=\bigcup_{i=0}^{n_{2 k}-1} f^{i}\left(\Delta_{2 k}\right) .
$$

By construction, the Cantor set $\Phi(f)$ is minimal, maximal transitive, and Lyapunov stable for $f$ and $f^{-1}$, see Proposition 4.2 and Section 4 for the details of this construction

A property $\mathscr{P}$ of a diffeomorphism $f$ is a robust property if every diffeomorphism in some $\mathrm{C}^{1}$-neighborhood of $f$ verifies $\mathscr{P}$.

Similarly, a property $\mathscr{P}$ is generic in an open set $\mathscr{V}$ of diffeomorphisms if there is a residual subset $\mathscr{R}$ of $\mathscr{V}$ consisting of diffeomorphisms satisfying $\mathscr{P}$. Finally, a property $\mathscr{P}$ is locally generic if it is generic in some non-empty open set of $\operatorname{Diff}^{\mathrm{l}}(\mathrm{M})$. As a direct consequence of Theorem $\mathrm{B}$ we now have the following heuristic principle.

Heuristic principle. - Given any robust or locally generic property $\mathscr{P}$ of $\operatorname{Diff}_{\text {int }}^{+}\left(\mathrm{D}^{3}\right)$ there is a residual subset $\mathscr{R}_{\mathscr{P}}(\mathrm{M})$ of $\mathscr{W}_{0}(\mathrm{M})$ of diffeomorphisms satisfying $\mathscr{P}$. Moreover, this property is displayed by every $f \in \mathscr{R}_{\mathscr{P}}(\mathrm{M})$ in infinitely many disjoint periodic disks.

In the next corollary we summarize some of these dynamical robust properties we consider more relevant: 
Corollary C. - There is a residual subset $\mathscr{R}(\mathrm{M})$ of the set $\mathscr{W}_{0}(\mathrm{M})$ contained in the set of wild diffeomorphisms consisting of diffeomorphisms $f$ having simultaneously infinitely many:

a) Sinks and sources.

b) Independent saddles, (i.e., saddles whose homoclinic classes consist only of a periodic orbit).

c) Non-trivial uniformly hyperbolic transitive attractors and repellors.

d) Non-trivial partially hyperbolic robustly transitive attractors and repellors.

e) Homoclinic classes containing persistently saddles of different indices.

f) Wild homoclinic classes $\Lambda_{i} \in \mathrm{W}_{0}(f)$.

g) Aperiodic maximal transitive Cantor sets $\mathscr{F}_{i}$ where $f$ is minimal. Moreover, the sets $\mathscr{F}_{i}$ are simultaneously Lyapunov stable for $f$ and $f^{-1}$, hence every $\mathscr{F}_{i}$ is saturated.

Moreover, the wild homoclinic classes $\Lambda_{i}$ and the aperiodic maximal transitive sets $\mathscr{F}_{i}$ can be chosen such that

$$
\overline{\bigcup \Lambda_{i}}=\overline{\bigcup \mathscr{F}_{i}}
$$

Let us observe that we do not know if last the equality in the corollary holds for all the aperiodic maximal transitive sets.

Our constructions can be carried out in higher dimensions. First, a trivial way to get aperiodic maximal transitive sets in higher dimensions consists of multiplying the dynamics on $\mathrm{D}^{3}$ by a hyperbolic transverse dynamics in such a way the disk turns out to be normally hyperbolic. On the other hand, a more interesting approach is to get universal dynamics in higher dimensions by defining wild homoclinic classes in $\mathrm{W}_{0}(f)$ similarly: these classes contain in a robust way periodic saddles of any possible index $(2, \ldots, \operatorname{dim}(\mathrm{M})-1)$, possess complex (non-real) eigenvalues of any rank (see [BDP] for the definition of rank), and, for any $k \in[2, \operatorname{dim} \mathrm{M}]$, has homoclinically related points of index $k$ having Jacobians greater than and less than one.

Recall that a topological attractor of a diffeomorphism $f$ is a compact set $\Gamma$ such that there is a compact neighbourhood $\Delta$ of it such that $f(\Delta)$ is contained in the interior of $\Delta$ and $\cap_{i \geq 0} f^{i}(\Delta)=\Gamma$. By construction, the aperiodic maximal transitive sets in Corollary $\mathrm{C}$ are a countable intersection of (nontransitive) attractors. Thus, in the terminology introduced in $[\mathrm{Hu}]$, these sets are quasi-attractors. Recall that the aperiodic maximal transitive Cantor sets here are Lyapunov stable for $f$ and $f^{-1}$, so they have trivial stable and unstable basins. This fact gives counter-examples ${ }^{2}$ (in the $\mathrm{C}^{1}$-setting) to the following question posed in $[\mathrm{Hu}]$ :

\footnotetext{
${ }^{2}$ This consequence of the results in this paper has been pointed out to us by M.-C. Arnaud and by a referee.
} 
Are generic chain recurrent quasi-attractors attractors?

In the case of tame diffeomorphisms, i.e., diffeomorphisms having finitely many pairwise disjoint homoclinic classes and whose number is locally generically constant, in $[\mathrm{CM}]$ it is proved that the union of the basins of the (topological) attractors is open and dense in the ambient manifold. This result, the question in $[\mathrm{Hu}]$, and our constructions (in the world of the wild diffeomorphisms) suggest the following problems concerning the size of the basins of attraction of the aperiodic maximal transitive sets with minimal dynamics in this paper.

Problems.

- Consider a diffeomorphism $f$ in the residual subset $\mathscr{R}(\mathrm{M})$ of $\mathscr{W}_{0}(\mathrm{M})$ in Corollary C. Is there an aperiodic maximal transitive set $\mathscr{F}$ of $f$ whose basin of attraction has non-empty interior? Or a weaker version of this question; does the basin of attraction of $\mathscr{F}$ contain a residual subset in some non-empty open subset of M?

- In the opposite direction of the previous questions, there is the following natural problem. For generic diffeomorphisms $f$ of $\operatorname{Diff}^{1}(\mathrm{M})$, is the union of the basins of the (topological) transitive attractors of $f$ dense in the whole manifold? For generic diffeomorphisms $f$, the set of periodic points of $f$ is dense in the nonwandering set of $f$, in particular any topological attractor of $f$ contains periodic points. Hence, a positive answer to this problem will imply a negative answer to the first one.

This paper is organized as follows. In the next section we sketch the proof of Theorem B. In Section 3 we give the complete proof of Theorem B. This section has two preparatory parts, the first one concerning perturbations of the identity (see Section 3.1) and the second one about dynamical perturbations of the derivative (see Section 3.2). In Section 4 we deduce Theorem A from Theorem B. Corollary G is proved in Section 5. Finally, in the Appendix (Section 6) we outline the construction of the wild homoclinic classes in $\mathbf{W}_{0}(f)$.

\section{Sketch of the proof of Theorem B}

Consider an open subset $\mathscr{W}_{0}$ of $\mathscr{W}_{0}(\mathrm{M})$ of diffeomorphisms $f$ having a wild homoclinic class $\mathrm{H}\left(\mathrm{P}_{f}, f\right) \in \mathrm{W}_{0}(f)$, where $\mathrm{P}_{f}$ is a saddle of index 2 depending continuously on $f \in \mathscr{W}_{0}$. Observe that $\mathscr{W}_{0}(\mathrm{M})$ can be written as union of the sets of the form of $\mathscr{W}_{0}$, so that it is enough to prove Theorem $\mathrm{B}$ for the set $\mathscr{W}_{0}$.

The proof of Theorem B consists of the following steps:

Step 1. - We begin by proving that given any open subset $\mathscr{O}$ of $\operatorname{Diff}_{\text {int }}^{+}\left(\mathrm{D}^{3}\right)$ and any open set $\mathrm{V}$ of $\mathrm{M}$, there are an arbitrarily small $\mathrm{C}^{1}$-perturbation $h$ of the 
identity map in $\mathrm{V}$, a small disk $\mathrm{D} \subset \mathrm{V}$, and $k>0$ such that the $(k-1)$ first iterates of $\mathrm{D}$ by $h$ are pairwise disjoint, $h^{k}(\mathrm{D}) \subset \operatorname{int}(\mathrm{D})$, and $\left.h^{k}\right|_{\mathrm{D}}$ is differentially conjugate to some $g \in \mathscr{O}$. This is done in Proposition 3.1.

Given $f \in \mathscr{W}_{0}$ and $\varepsilon>0$ consider the set $\Sigma(\varepsilon, f)$ of hyperbolic periodic points $\mathrm{R}$ of $f$ such that

- $\mathrm{R}$ is homoclinically related to $\mathrm{P}_{f}$, and

- $\frac{\log \left(\operatorname{Jac}_{f \operatorname{per}(\mathrm{R})}(\mathrm{R})\right)}{\operatorname{per}(\mathrm{R})} \in(-\varepsilon, \varepsilon)$.

Step 2. - The next step is to see that, for every $\varepsilon>0$ and every $f \in \mathscr{W}_{0}$, the set $\Sigma(\varepsilon, f)$ is dense in $\mathrm{H}\left(\mathrm{P}_{f}, f\right)$, see Lemma 3.5.

Step 3. - Using the point $\mathbf{Q}_{f}$ of index one with a non-real expanding eigenvalue in the definition of wild homoclinic class in $\mathrm{W}_{0}(f)$, we have that $\mathrm{H}\left(\mathrm{P}_{f}, f\right)$ does not admit any dominated splitting for all $f \in \mathscr{W}_{0}$. Step 2 now implies that, for every $f \in \mathscr{W}_{0}$ and every $\varepsilon>0$, the set $\Sigma(\varepsilon, f)$ does not admit any dominated splitting (see Definition 3.7 and Lemma 3.8).

Step 4. - Using the fact that $\Sigma(\varepsilon, f)$ does not admit any dominated splitting, Proposition 2.1 in [BDP] implies that, for every $\delta>0$, there are a point $\mathrm{P}_{1} \in$ $\Sigma(\varepsilon, f)$ and a $\delta$-perturbation of the derivative of $f$ throughout the orbit of $\mathrm{P}_{1}$ (without modifying neither the $f$-orbit of $\mathrm{P}_{1}$ nor the Jacobian of $f$ at $\mathrm{P}_{1}$ ) such that the linear map $\mathrm{A}: \mathrm{T}_{\mathrm{P}_{1}} \mathrm{M} \mapsto \mathrm{T}_{\mathrm{P}_{1}} \mathrm{M}$ corresponding to the product of the perturbed derivatives along the orbit of $\mathrm{P}_{1}$ is a homothety. By definition of $\Sigma(\varepsilon, f)$, the linear map A verifies

$$
\frac{\log (\operatorname{det}(\mathrm{A}))}{\operatorname{per}\left(\mathrm{P}_{1}\right)} \in[-\varepsilon, \varepsilon] .
$$

So, after a new $\varepsilon$-perturbation, we can assume that $\operatorname{det}(\mathrm{A})=1$, i.e., the linear map $\mathrm{A}$ is the identity.

Step 5. - By a lemma of Franks (see Lemma 3.4), we can perform the previous perturbation of the derivative of $f$ dynamically, obtaining a diffeomorphism $g$ arbitrarily $\mathrm{C}^{1}$-close to $f$ with a periodic point $\mathrm{P}_{1}$ whose derivative is the identity. After a new small perturbation, if necessary, we can assume that $g^{\operatorname{per}\left(\mathrm{P}_{1}\right)}$ is the identity map in a small neighborhood $\mathrm{V}$ of $\mathrm{P}_{1}$, see Theorem 3.2.

Step 6. - The end of the proof of Theorem B involves an inductive argument (see Section 3.3). In rough terms, we apply Step 1 to $g^{\operatorname{per}\left(\mathrm{P}_{1}\right)}$ and check that the disk $\mathrm{D}$ in Step 1 can be taken arbitrarily close to any point $x \in \mathrm{H}\left(\mathrm{P}_{f}, f\right)$. This will be done using the density of $\Sigma(\varepsilon, f)$ in $\mathrm{H}\left(\mathrm{P}_{f}, f\right)$ and the constructions in $[\mathrm{BDP}]$. Finally, we get in the disk $\mathrm{D}$ a point with a wild homoclinic class and repeat the procedure above (this gives the inductive pattern). 


\section{Proof of Theorem B}

\subsection{Perturbations of the identity map}

Proposition 3.1. - Let $\mathrm{D} \subset \mathrm{M}$ be a compact ball and $\phi$ any diffeomorphism in Diff $_{\text {int }}^{+}\left(\mathrm{D}^{3}\right)$. Then every neighborhood of the identity map of $\mathrm{D}$ contains a diffeomorphism $f$ such that

- $f$ coincides with the identity in a neighborhood of the boundary of $\mathrm{D}$,

- there are a disk $\mathrm{D}_{0} \subset \mathrm{D}$ and a natural number $n_{0}$ such that $\mathrm{D}_{0}, f\left(\mathrm{D}_{0}\right), \ldots, f^{n_{0}-1}\left(\mathrm{D}_{0}\right)$ are pairwise disjoint sets, $f^{n_{0}}\left(\mathrm{D}_{0}\right) \subset \operatorname{int}\left(\mathrm{D}_{0}\right)$, and the restriction of $f^{n_{0}}$ to $\mathrm{D}_{0}$ is differentially conjugate to some $\phi \in \mathscr{O}$.

This proposition follows from the following classical result (see J. Cerf, [Ce]):

Theorem. - Let $\operatorname{Diff}_{0}^{1}\left(\mathrm{D}^{3}\right)$ be the group of $\mathrm{C}^{1}$-diffeomorphisms of the disk $\mathrm{D}^{3}$ coinciding with the identity in a neighborhood of the boundary $\mathrm{\partial D}^{3}$ of $\mathrm{D}^{3}$. Then every diffeomorphism $g \in \operatorname{Diff}_{0}^{1}\left(\mathrm{D}^{3}\right)$ is isotopic to the identity by a path $g_{t} \in \operatorname{Diff}_{0}^{1}\left(\mathrm{D}^{3}\right)$.

Proof of the proposition. - Observe first that, up to a choice of a diffeomorphism $\psi: \mathrm{D} \rightarrow \mathrm{D}^{3}$, one can assume that $\mathrm{D}$ is equal to $\mathrm{D}^{3}$.

Fix $\phi$ in Diff $_{\text {int }}^{+}\left(\mathrm{D}^{3}\right)$ and choose a diffeomorphism $g$ of $\mathrm{D}^{3}$ coinciding with the identity in a neighborhood of the boundary of $\mathrm{D}^{3}$ and such that there is a disk $\hat{\mathrm{D}}$ in the interior of $\mathrm{D}^{3}$ such that the restriction of $g$ to $\hat{\mathrm{D}}$ is smoothly conjugate to $\phi$.

The previous theorem implies that $g$ can be written as the composition $g_{k} \circ$ $\cdots \circ g_{1} \circ g_{0}$ of finitely many diffeomorphisms $g_{i}$ arbitrarily close to the identity.

We first perturb the identity map of $\mathrm{D}^{3}$ to get a diffeomorphism $h$ equal to identity in a neighborhood of the boundary $\partial \mathrm{D}^{3}$, and a periodic round disk $\mathrm{D}_{0} \subset \mathrm{D}^{3}$ of arbitrarily large period $n_{0} \geq k$ such that

- $\mathrm{D}_{0}, h\left(\mathrm{D}_{0}\right), \ldots, h^{n_{0}-1}\left(\mathrm{D}_{0}\right)$ are pairwise disjoint,

- $h: h^{i}\left(\mathrm{D}_{0}\right) \rightarrow h^{i+1}\left(\mathrm{D}_{0}\right)$ is an isometry for every $i \in\left\{0, \ldots, n_{0}-1\right\}$,

- $h^{n_{0}}: \mathrm{D}_{0} \rightarrow \mathrm{D}_{0}$ is equal to the identity.

We now write $g=g_{n_{0}-1} \circ \cdots \circ g_{0}$, where, for $0 \leq i \leq k$, the $g_{i}$ are as above and, for $i>k$, we take $g_{i}$ equal to the identity. Consider an affine bijective map $\mathrm{H}: \mathrm{D}_{0} \rightarrow \mathrm{D}^{3}$, and define

$$
\tilde{g}_{i}=\mathrm{H}^{-1} \circ g_{i} \circ \mathrm{H}: \mathrm{D}_{0} \rightarrow \mathrm{D}_{0} .
$$

Replace now the restriction of $h$ to each disk $h^{i}\left(\mathrm{D}_{0}\right)$ by the map $h^{i+1} \circ \tilde{g}_{i-1} \circ$ $h^{-i}$ and denote by $f$ the resulting diffeomorphism. Since the restrictions of $h$ to the iterates $h^{i}\left(\mathrm{D}_{0}\right)$ are isometries and the maps $g_{i}$ are $\mathrm{C}^{1}$-close to identity, the 
diffeomorphism $f$ is $\mathrm{C}^{1}$-close to $h$. Observe that, by construction, the restriction of $f^{n_{0}}$ to $\mathrm{D}_{0}$ is

$$
f^{n_{0}}=h^{n_{0}} \circ \tilde{g}_{n_{0}-1} \circ \cdots \circ \tilde{g}_{0}=\mathrm{H}^{-1} \circ g_{n_{0}-1} \circ \cdots \circ g_{0} \circ \mathrm{H}=\mathrm{H}^{-1} \circ g \circ \mathrm{H},
$$

where the second identity follows recalling that the restriction of $h^{n_{0}}$ to $\mathrm{D}_{0}$ is the identity. Since $g$ is differentially conjugate to $\phi$, it is clear that the disk $\mathrm{D}_{0}$, the natural number $n_{0}$, and the map $f$ verify all properties in the proposition, ending the proof of the proposition.

\subsection{Perturbations of the derivative at a periodic point}

Theorem 3.2. - Consider the open subset $\mathscr{W}_{0}$ of $\mathscr{W}_{0}(\mathrm{M})$ of diffeomorphisms $f$ having a wild homoclinic class $\mathrm{H}\left(\mathrm{P}_{f}, f\right) \in \mathrm{W}_{0}(f)$, where the point $\mathrm{P}_{f}$ has index 2 and depends continuously on $f \in \mathscr{W}_{0}$. Then for every diffeomorphism $f$ in $\mathscr{W}_{0}$ and every $\varepsilon>0$ there are:

- a hyperbolic periodic point $x$ homoclinically related to $\mathrm{P}_{f}$ (thus $x \in \mathrm{H}\left(\mathrm{P}_{f}, f\right)$ ) whose orbit is $\varepsilon$-dense in $\mathrm{H}\left(\mathrm{P}_{f}, f\right)$,

- a small neighborhood $\mathrm{V}$ of $x$ and an $\varepsilon$-perturbation $g$ of $f$ along the orbit of $x$, such that the sets $\mathrm{V}, g(\mathrm{~V}), \ldots, g^{p(x)-1}(\mathrm{~V})$ are pairwise disjoint and the restriction of $g^{p(x)}$ to $\mathrm{V}$ is the identity map $(p(x)$ is the period of $x)$. Moreover, the orbits of $x$ by $f$ and $g$ coincide.

Given a periodic point $x$ of $f$ we let

$$
\mathrm{J}(x, f)=\frac{\log \left(\operatorname{Det}\left(\mathrm{D} f^{p(x)}(x)\right)\right)}{p(x)} .
$$

For given $\varepsilon>0$ and $f \in \mathscr{W}_{0}$, denote by $\Sigma(\varepsilon, f)$ the set of periodic points $x$ homoclinically related to $\mathrm{P}_{f}$ whose orbit is $\varepsilon$-dense in $\mathrm{H}\left(\mathrm{P}_{f}, f\right)$ and such that $\mathrm{J}(x, f) \in(-\varepsilon, \varepsilon)$.

To prove Theorem 3.2 we analyze the linear cocycle defined by the differential of $f$ over the wild homoclinic class $\mathrm{H}\left(\mathrm{P}_{f}, f\right)$, its restriction to $\Sigma(\varepsilon, f)$, and the perturbations of this cocycle. A lemma of Franks (Lemma 3.4) will allow us to realize such perturbations of the cocycle as $\mathrm{C}^{1}$-perturbations of $f$. The main step to prove Theorem 3.2 is now the following proposition:

Proposition 3.3. - Let $\mathscr{W}_{0} \subset \mathscr{W}_{0}(\mathrm{M})$ as in Theorem 3.2. Then for every $f \in \mathscr{W}_{0}$ and every $\varepsilon>0$ there are a hyperbolic periodic point $x$ in $\Sigma(\varepsilon, f)$ and an $\varepsilon$-perturbation

$$
\mathrm{A}\left(f^{i}(x)\right): \mathrm{T}_{f^{i}(x)} \mathrm{M} \rightarrow \mathrm{T}_{f^{i+1}(x)} \mathrm{M}
$$

of the derivative of $\mathrm{D} f$ along the orbit of $x$ such that the product

$$
\mathrm{M}_{\mathrm{A}}(x)=\mathrm{A}\left(f^{p(x)-1}\right) \circ \cdots \circ \mathrm{A}(x)
$$

is the identity map. 
Before proving the proposition, let us prove Theorem 3.2.

Proof of the theorem. — We begin by stating the announced lemma of Franks:

Lemma 3.4 ([Fr], [Ma]). - Consider a $\mathrm{C}^{1}$-diffeomorphism $\varphi$ and a $\varphi$-invariant finite set $\Sigma$. Let $\mathrm{L}$ be an $\varepsilon$-perturbation of $\mathrm{D} \varphi$ along $\Sigma$ (i.e., the linear maps $\mathrm{L}(x)$ and $\mathrm{D} \varphi(x)$ are $\varepsilon$-close for all $x \in \Sigma$ ). Then for every neighborhood $\mathrm{U}$ of $\Sigma$ there is a diffeomorphism $\phi$, $\mathrm{C}^{1}-\varepsilon$-close to $\varphi$, such that:

- $\varphi(x)=\phi(x)$ if $x \in \Sigma$ or if $x \notin \mathrm{U}$,

- $\mathrm{D} \phi(x)=\mathrm{L}(x)$ for all $x \in \Sigma$.

Applying Lemma 3.4 to a diffeomorphism $f$ in $\mathscr{W}_{0}$, the set $\Sigma=\{x, f(x)$, $\left.\ldots, f^{p(x)-1}(x)\right\}$ ( $x$ as in Proposition 3.3), and the linear map $\mathrm{L}=\mathrm{A}$ (A is the perturbation of the derivative in Proposition 3.3), we get a perturbation $g$ of $f$ preserving the orbit of $x$ with $\mathrm{D} g^{p(x)}(x)=\mathrm{I} d$.

The first part of the theorem follows recalling that, by definition of $\Sigma(\varepsilon, f)$, the orbit of $x$ is $\varepsilon$-dense in $\mathrm{H}\left(\mathrm{P}_{f}, f\right)$.

For the second part of the theorem, observe that, after a new perturbation of $g$ throughout the orbit of $x$, we get a neighborhood $\mathrm{V}$ of $x$ such that $\mathrm{V}, g(\mathrm{~V})$ and $g^{p(x)-1}(\mathrm{~V})$ are pairwise disjoint and the restriction of $g^{p(x)}$ to $\mathrm{V}$ is the identity. Observe that, by construction, the orbits of $x$ by $f$ and $g$ are the same.

The proof of Theorem 3.2 is now complete.

The proof of Proposition 3.3 is a small variation of the main technical result in [BDP]: Given any non-trivial homoclinic class $\mathrm{H}$ that does not admit any dominated splitting (see Definition 3.7) there are a periodic point $x \in \mathrm{H}$ and a perturbation of the derivative of $f$ along the orbit of $x$ such that the product of the perturbed derivatives along this orbit is a homothety, (see [BDP, Proposition 2.1]). Here we use the fact that the points in $\Sigma(\varepsilon, f)$ have Jacobians close to 1 to get the homothety being the identity. The main difficulty here is to check that the set $\Sigma(\varepsilon, f)$ verifies the hypotheses in $[\mathrm{BDP}]$.

To prove Proposition 3.3 we need to state some properties of the sets $\Sigma(\varepsilon, f)$. This will be done in the next two lemmas. $\mathrm{H}\left(\mathrm{P}_{f}, f\right)$.

Lemma 3.5. - Consider any $\varepsilon>0$ and $f \in \mathscr{W}_{0}$. Then $\Sigma(\varepsilon, f)$ is a dense subset of

Proof. - Recall that, by definition of wild homoclinic class in $\mathrm{W}_{0}(f)$, we have that $\mathrm{J}\left(\mathrm{P}_{f}, f\right)>1$ and that $\mathrm{H}\left(\mathrm{P}_{f}, f\right)$ contains a point $\mathrm{P}_{f}^{\prime}$ homoclinically related to $\mathrm{P}_{f}$ with $\mathrm{J}\left(\mathrm{P}_{f}^{\prime}, f\right)<1$. This implies that for every $\delta>0$ there is a hyperbolic 
basic set ${ }^{3} \mathrm{H}_{\delta} \subset \mathrm{H}\left(\mathrm{P}_{f}, f\right)$ containing $\mathrm{P}_{f}$ and $\mathrm{P}_{f}^{\prime}$ which is $\delta$-dense in $\mathrm{H}\left(\mathrm{P}_{f}, f\right)$. We choose a Markov partition of $\mathrm{H}_{\delta}$ by rectangles of small size such that the variation of $\log (\operatorname{det}(\mathrm{D} f(x))$ in each rectangle is less than $\varepsilon / 2$.

Now, given any periodic point $x \in \mathrm{H}_{\delta}, \mathrm{J}(x, f)$ is determined (up to an error of $\varepsilon / 2$ ) by its itinerary. For each $n_{1}$ and $n_{2}$ (big enough) there is a periodic point $x$ of period $n_{1}+n_{2}+k$ spending $n_{1}$ consecutive iterates in the same rectangles that the iterates of $\mathrm{P}_{f}, n_{2}$ consecutive iterates in the rectangle containing the iterates of $\mathrm{P}_{f}^{\prime}$, and $k$ iterates passing through all rectangles of the partition, where $k$ is bounded independently of $n_{1}$ and $n_{2}$. Choosing appropriated $n_{1}$ and $n_{2}$, we get a point $x \in \mathrm{H}_{\delta}$ such that $\mathrm{J}(x, f)$ is $\varepsilon$-close to 0 and whose orbit intersects all the rectangles of the partition. As we can take the size of the rectangles of the partition and $\delta$ arbitrarily small, we get that the orbit of $x$ is $\varepsilon$-dense in $\mathrm{H}\left(\mathrm{P}_{f}, f\right)$. Thus the periodic point $x$ belongs to $\Sigma(\varepsilon, f)$. The same proof gives the density of $\Sigma(\varepsilon, f)$ in $\mathrm{H}\left(\mathrm{P}_{f}, f\right)$. This ends the proof of the lemma.

We have the following lemma which follows straightforwardly from the proof of Lemma 3.5:

Lemma 3.6. - Consider any $\varepsilon>0$ and $f \in \mathscr{W}_{0}$. Then given any finite subset $\Xi$ of $\Sigma(\varepsilon, f)$ there is a basic transitive set $\Lambda$ containing $\Xi$ whose periodic points belong to $\Sigma(\varepsilon, f)$.

Proof. - Observe that all the points in the set $\Xi$ are homoclinically related and, for every $x \in \Xi,|\mathrm{J}(x, f)|<(\varepsilon-\delta)$ for some $\delta>0$. Thus there is a basic set $\Lambda^{\prime} \subset \mathrm{H}\left(\mathrm{P}_{f}, f\right)$ containing $\Xi$. Consider now a Markov partition of $\Lambda^{\prime}$ by sufficiently small rectangles (as in Lemma 3.5, this allows us to control the Jacobians up to an error less than $\delta / 2)$. Finally, the set $\Lambda$ corresponds to the points in $\Lambda^{\prime}$ whose itineraries are determined by a sub-shift of finite type associated to the rectangles of the partition. As in Lemma 3.5, the choice of the sub-shift is done to have a suitable control of the Jacobians. The proof of the lemma is now complete.

Let us recall the definition of dominated splitting.

Definition 3.7. - A dominated splitting of an $f$-invariant set $\Lambda$ is a $\mathrm{D}$ f-invariant splitting $\mathrm{E} \oplus \mathrm{F}$ defined over $\mathrm{T}_{\Lambda} \mathrm{M}$ such that the fibers of $\mathrm{E}$ and $\mathrm{F}$ have constant dimension and there is $k \geq 1$ such that for every $x \in \Lambda$ one has

$$
\|\left.\left.\mathrm{D} f^{k}\right|_{\mathrm{E}(x)}|| \cdot|| \mathrm{D} f^{-k}\right|_{\mathrm{F}\left(f^{k}(x)\right)}||<\frac{1}{2},
$$

that is, the derivative $\mathrm{D} f$ expands the vectors in $\mathrm{F}$ uniformly more than the vectors in $\mathrm{E}$. Then we say that $\mathrm{F}$ dominates $\mathrm{E}$ and write $\mathrm{E} \prec \mathrm{F}$.

${ }^{3}$ A basic set is a transitive hyperbolic compact set with local product structure, or, equivalently, which is the maximal invariant set in some neighborhood of it. 
Lemma 3.8. - Consider any diffeomorphism $f \in \mathscr{W}_{0}$ and $\varepsilon>0$. Then the set $\Sigma(\varepsilon, f)$ does not admit any dominated splitting.

Proof. - By Lemma 3.5, the set $\Sigma(\varepsilon, f)$ is dense in $\mathrm{H}(\mathrm{P}, f)$. Observing that a dominated splitting defined in a set $\Lambda$ always admits a dominated extension to the closure of $\Lambda$ (see, for instance, [BDP, Lemma 1.4]), it is enough to see that $\mathrm{H}\left(\mathrm{P}_{f}, f\right)$ does not admit any dominated splitting for every $f \in \mathscr{W}_{0}$.

We argue by contradiction, let $f \in \mathscr{W}_{0}$ and suppose that there is a dominated splitting

$$
\mathrm{T}_{\mathrm{H}\left(\mathrm{P}_{f}, f\right)} \mathrm{M}=\mathrm{E} \oplus \mathrm{F}, \quad \mathrm{E} \prec \mathrm{F} .
$$

The fact that $\mathrm{P}_{f}$ has a non-real contracting eigenvalue implies that $\operatorname{dim}(\mathrm{E})=2$. Similarly, the fact that $\mathrm{Q}_{f} \in \mathrm{H}\left(\mathrm{P}_{f}, f\right)$ has a non-real expanding eigenvalue implies that $\operatorname{dim}(\mathrm{F})=2$. Thus $\operatorname{dim}(\mathrm{E})+\operatorname{dim}(\mathrm{F})=4>3=\operatorname{dim}(\mathrm{M})$, which is a contradiction.

We can now finish the proof of Proposition 3.3:

Proof of the proposition. - Let $f \in \mathscr{W}_{0}$. Consider the continuous linear cocycle $\mathscr{D}$ induced by $\mathrm{D} f$ over $\Sigma(\varepsilon, f)$. By Lemma 3.8, this cocycle does not admit any dominated splitting. As all orbits in $\Sigma(\varepsilon, f)$ are periodic, we have a continuous linear periodic system, according to the terminology in [BDP, Section 1.1].

Lemma 3.9 and the proof of [BDP, Lemma 1.9] imply that the cocycle $\mathscr{D}$ admits transitions, see [BDP, Definitions 1.6 and 1.8 $]^{4}$. This implies that the cocycle $\mathscr{D}$ over $\Sigma(\varepsilon, f)$ verifies the hypotheses of [BDP, Proposition 2.1]. So, given any $\delta>0$, there are a point $x \in \Sigma(\varepsilon, f)$ and a $\delta$-perturbation $\mathscr{B}$ of $\mathscr{D}$ along the orbit of $x$ such that $\mathrm{M}_{\mathrm{B}}(x)$ is a homothety (recall that $\mathrm{M}_{\mathrm{B}}(x)=\mathrm{B}\left(f^{p(x)-1}\right) \circ \cdots \circ \mathrm{B}(x)$ ). Moreover, if $\delta$ is small enough,

$$
\mathrm{J}(x, \mathscr{B})=\frac{\log \left(\operatorname{det}\left(\mathrm{M}_{\mathrm{B}}(x)\right)\right)}{p(x)} \in[-2 \varepsilon, 2 \varepsilon] .
$$

By multiplying $\mathrm{B}$ along the orbit of $x$ by a number $\lambda$ close to 1 (in fact, $\lambda=$ $e^{-|\mathrm{J}(x, \mathscr{B})| / 3}$, where $\left.3=\operatorname{dim}(\mathrm{M})\right)$ we get a new perturbation $\mathscr{A}$ of $\mathscr{D}$ along the orbit of $x$ such that $\mathrm{M}_{\mathrm{A}}(x)=\mathrm{I} d$. This ends the proof of the proposition.

${ }^{4}$ The precise definition of transitions is somewhat technical and we do not use explicitly it here. This notion is a formalization of the following intuitive idea: consider a finite set $\mathrm{X}$ of periodic orbits of a hyperbolic basic set $\Lambda$. Then, there are periodic points of $\Lambda$ whose orbits visit a small neighborhood of some orbit of X, remain there an arbitrarily large time and go (in a uniformly bounded time) to the neighborhood of another orbit of X, remain there an arbitrarily large time, and so on, until the period. The derivatives at these orbits are essentially products of the derivatives at the orbits of $\mathrm{X}$ with some correcting terms, which are bounded matrices called transitions and correspond to the itineraries out of a small neighborhood of X. Here Lemma 3.9 asserts that any finite set of $\Sigma(\varepsilon, f)$ is contained in a basic set whose periodic orbits are in $\Sigma(\varepsilon, f)$, providing the existence of transitions. 


\subsection{End of the proof of Theorem $B$}

Given an open subset $\mathscr{O}$ of $\operatorname{Diff}_{\text {int }}^{+}\left(\mathrm{D}^{3}\right)$ and $\varepsilon>0$ let $\mathscr{P}\left(\mathscr{O}, \varepsilon, \mathscr{W}_{0}\right)$ be the subset of $\mathscr{W}_{0}\left(\mathscr{W}_{0}\right.$ is the open subset of $\mathscr{W}_{0}(\mathrm{M})$ in Theorem 3.2) of diffeomorphisms $f$ such that there are a neighborhood $\mathscr{U}_{f}$ of $f$ in $\mathscr{W}_{0}$, a disk $\mathrm{D}$, and a natural number $n>0$, such that every $g \in \mathscr{U}_{f}$ satisfies the following properties:

$(\mathscr{P} \mathbf{1})$ the iterates $g^{i}(\mathrm{D}), i=0, \ldots, n-1$, are pairwise disjoint,

$(\mathscr{P} \mathbf{2}) g^{n}(\mathrm{D})$ is contained in the interior of $\mathrm{D}$,

( $\mathscr{P} 3)$ for every point $x \in \mathrm{H}\left(\mathrm{P}_{g}, g\right)$ there is $i \in\{0, \ldots, n-1\}$ such that $g^{i}(\mathrm{D})$ is contained in the ball $\mathrm{B}(x, \varepsilon)$,

$\left(\mathscr{P} \mathbf{3}^{\text {}}\right)$ the iterates $g^{i}(\mathrm{D}), i=0, \ldots, n-1$, are contained in the $\varepsilon$-neighborhood of $\mathrm{H}\left(\mathrm{P}_{g}, g\right)$,

$(\mathscr{P} \mathbf{4})$ the diameters of the family of disks $\left\{g^{i}(\mathrm{D})\right\}_{i=-n}^{n-1}$ are upper bounded by $2 \varepsilon$,

$(\mathscr{P} \mathbf{5})$ the restriction of $g^{n}$ to $\mathrm{D}$ is differentially conjugate to some $\varphi \in \mathscr{O}$.

We denote by $\mathscr{P}^{-}\left(\mathscr{O}, \varepsilon, \mathscr{W}_{0}\right)$ the set of diffeomorphisms $f$ in $\mathscr{W}_{0}$ whose inverse $f^{-1}$ belongs to $\mathscr{P}\left(\mathscr{O}, \varepsilon, \mathscr{W}_{0}\right)$.

\section{Remark 3.9.}

1. The sets $\mathscr{P}\left(\mathscr{O}, \varepsilon, \mathscr{W}_{0}\right)$ and $\mathscr{P}^{-}\left(\mathscr{O}, \varepsilon, \mathscr{W}_{0}\right)$ are both open (even if the property $\mathscr{P} 3$ is not open): by definition, any $f \in \mathscr{P}\left(\mathscr{O}, \varepsilon, \mathscr{W}_{0}\right)$ admits a neigh-

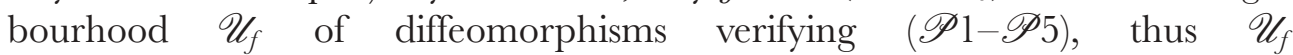
$\subset \mathscr{P}\left(\mathscr{O}, \varepsilon, \mathscr{W}_{0}\right)$.

A priori, these sets may be empty. In the next lemma we will see that they are dense in $\mathscr{W}_{0}$.

2. For every $f \in \mathscr{P}\left(\mathscr{O}, \varepsilon, \mathscr{W}_{0}\right)$, there is a neighborhood $\mathscr{V}$ of $f$ where the natural number $n$ is constant, and the disk $\mathrm{D}$ and the conjugacy between the restriction of $g^{n}$ to $\mathrm{D}$ and some element of $\mathscr{O} \subset \operatorname{Diff}_{\text {int }}^{+}\left(\mathrm{D}^{3}\right)$ can be chosen depending continuously on $g$. We call this conjugacy the identification between $\mathrm{D}$ and $\mathrm{D}^{3}$.

Lemma 3.10. - The sets $\mathscr{P}\left(\mathscr{O}, \varepsilon, \mathscr{W}_{0}\right)$ and $\mathscr{P}^{-}\left(\mathscr{O}, \varepsilon, \mathscr{W}_{0}\right)$ are open and dense in $\mathscr{W}_{0}$ for every $\varepsilon>0$ and every open set $\mathscr{O} \subset \operatorname{Diff}_{\text {int }}^{+}\left(\mathrm{D}^{3}\right)$.

Proof. - We prove the lemma for $\mathscr{P}\left(\mathscr{O}, \varepsilon, \mathscr{W}_{0}\right)$, the proof for $\mathscr{P}^{-}\left(\mathscr{O}, \varepsilon, \mathscr{W}_{0}\right)$ is the same. By Remark 3.9, it is enough to prove the density of $\mathscr{P}\left(\mathscr{O}, \varepsilon, \mathscr{W}_{0}\right)$ in $\mathscr{W}_{0}$.

Observe that the map $g \mapsto \mathrm{H}\left(\mathrm{P}_{g}, g\right)$ is lower semi-continuous. So there is a residual subset $\mathscr{S}_{1} \subset \mathscr{W}_{0}$ where this map is continuous. Thus to prove the lemma it is enough to see that given any $f \in \mathscr{S}_{1}$ there is an arbitrarily small perturbation of $f$ in $\mathscr{P}\left(\mathscr{O}, \varepsilon, \mathscr{W}_{0}\right)$. 
Given $f \in \mathscr{S}_{1}$, consider $\varepsilon_{0}>0$ such that, for every diffeomorphism $g \varepsilon_{0}$-close to $f$, the Hausdorff distance between the homoclinic classes $\mathrm{H}\left(\mathrm{P}_{f}, f\right)$ and $\mathrm{H}\left(\mathrm{P}_{g}, g\right)$ is less than $\varepsilon / 10$. By Theorem 3.2, given any $0<\varepsilon_{1}<\inf \left\{\varepsilon_{0} / 10, \varepsilon / 10\right\}$, there are

- a hyperbolic periodic point $y \in \mathrm{H}\left(\mathrm{P}_{f}, f\right)$ homoclinically related to $\mathrm{P}_{f}$ whose orbit is $\varepsilon_{1}$-dense in $\mathrm{H}\left(\mathrm{P}_{f}, f\right)$,

- an $\varepsilon_{1}$-perturbation $f_{1}$ of $f$ along the orbit of $y$ and a neighborhood $\mathrm{V}$ of $y$ such that $\mathrm{V}, f_{1}(\mathrm{~V}), \ldots, f_{1}^{p(y)-1}(\mathrm{~V})$ are pairwise disjoint, the restriction of $f_{1}^{p(y)}$ to $\mathrm{V}$ is the identity, and the orbits of $y$ by $f$ and $f_{1}$ coincide.

Applying Proposition 3.1 to a small compact ball $\mathrm{D}$ contained in $\mathrm{V}$ and the open set $\mathscr{O}$, we get an $\varepsilon_{1}$-perturbation $\mathrm{F}$ of the identity in the disk $\mathrm{D}$ that can be extended to the identity outside the disk $\mathrm{D}$, a disk $\mathrm{D}_{0} \subset \mathrm{D}$ (with diameter less than $\varepsilon_{1}$ ) and $k$, such that the map $f_{2}=\mathrm{F} \circ f_{1}$ and the natural number $m=p(y) \cdot k$ satisfy the following properties:

(d1) the disks $\left\{f_{2}^{i}\left(\mathrm{D}_{0}\right)\right\}_{i=0}^{m-1}$ are pairwise disjoint,

(d2) $f_{2}^{m}\left(\mathrm{D}_{0}\right) \subset \operatorname{int}\left(\mathrm{D}_{0}\right)$ and the restriction of $f_{2}^{m}$ to $\mathrm{D}_{0}$ is differentially conjugate to an element of $\mathscr{O}$ (recall that $f_{1}^{p(y)}$ is the identity in $\mathrm{D}$, so $f_{2}^{p(y)}$ coincides with $\mathrm{F}$ in $\mathrm{D})$,

(d3) the disk $f_{2}^{i}\left(\mathrm{D}_{0}\right)$ is contained in the interior of the ball $\mathrm{B}\left(y_{i}, \varepsilon_{1}\right)$ of radius $\varepsilon_{1}$ centered at $y_{i}$, where $y_{i}=f_{1}^{i}(y)=f^{i}(y)$ and $i \geq 0$,

(d4) the diameters of the family of disks $\left\{f_{2}^{i}\left(\mathrm{D}_{0}\right)\right\}_{i=-m}^{m-1}$ are strictly upper bounded by $2 \operatorname{diam}\left(\mathrm{D}_{0}\right)=2 \varepsilon_{1}$.

Observe that conditions (d1), (d2), (d3) and (d4) are open ones.

The previous assertion implies that there is a non-empty open set $\mathscr{O}^{1}$ such that every $f_{2} \in \mathscr{O}^{1}$ is an $\varepsilon_{1}$-perturbation of $f_{1}$ and verifies (d1)-(d4) above, where in these conditions the disk $\mathrm{D}_{0}$, the natural number $m$, and the points $y_{i}$ do not depend on the diffeomorphisms, that is, the disks $\left\{f_{2}^{i}\left(\mathrm{D}_{0}\right)\right\}_{i=0}^{m-1}$ are pairwise disjoint, $f_{2}^{m}\left(\mathrm{D}_{0}\right) \subset \operatorname{int}\left(\mathrm{D}_{0}\right)$, the restriction of $f_{2}^{m}$ to $\mathrm{D}_{0}$ is differentially conjugate to an element of $\mathscr{O}$, and $f_{2}^{i}\left(\mathrm{D}_{0}\right)$ is contained in the interior of $\mathrm{B}\left(y_{i}, \varepsilon_{1}\right), i \in$ $\{0,1, \ldots, m-1\}$.

Let us state a remark about the disk $\mathrm{D}_{0}$ we will use later.

Remark 3.11. - The orbit of the disk $\mathrm{D}_{0}$ is disjoint from $\mathrm{H}\left(\mathrm{P}_{f_{2}}, f_{2}\right)$.

This remark follows by observing that the point $\mathrm{P}_{f_{2}}$ does not belong to the segment of orbit $\mathrm{V}, f_{2}(\mathrm{~V}), \ldots, f_{2}^{p(y)-1}(\mathrm{~V})$ and that the disk $\mathrm{D}_{0}$ is strictly forward $f_{2}$-invariant and contained in $\mathrm{V} \cup f_{2}(\mathrm{~V}) \cup \cdots \cup f_{2}^{p(y)-1}\left(\mathrm{~V}_{2}\right)$.

To conclude the proof of the lemma it is enough to check that every $f_{2} \in \mathscr{O}^{1}$ is an $\varepsilon_{0}$-perturbation of $f$ and belongs to $\mathscr{P}\left(\mathscr{O}, \varepsilon, \mathscr{W}_{0}\right)$. The first assertion follows immediately observing that $f_{2}$ is a $\left(3 \varepsilon_{1}\right)$-perturbation of $f$ and that $\varepsilon_{1}<\varepsilon_{0} / 10$. 
For the second one, observe that, by construction, $f_{2}$ verifies $(\mathscr{P} 1),(\mathscr{P} 2)$, $(\mathscr{P} 4)$ and $(\mathscr{P} 5)$ in the definition of $\mathscr{P}\left(\mathscr{O}, \varepsilon, \mathscr{W}_{0}\right)$. So it remains to see that $f_{2}$ also verifies $(\mathscr{P} 3)$ and $\left(\mathscr{P} 3^{\prime}\right)$.

Let us show that it verifies $(\mathscr{P} 3)$, that is, given any point $x_{2} \in \mathrm{H}\left(\mathrm{P}_{f_{2}}, f_{2}\right)$ there is $i \in\{0,1, \ldots, m-1\}$ such that $f_{2}^{i}\left(\mathrm{D}_{0}\right) \subset \mathrm{B}\left(x_{2}, \varepsilon\right)$.

To see why this is so observe that, by the choice of $\varepsilon_{0}$, the Hausdorff distance between $\mathrm{H}\left(\mathrm{P}_{f_{2}}, f_{2}\right)$ and $\mathrm{H}\left(\mathrm{P}_{f}, f\right)$ is less than $\varepsilon / 10$. Thus, given any $x_{2} \in$ $\mathrm{H}\left(\mathrm{P}_{f_{2}}, f_{2}\right)$ there is $x \in \mathrm{H}\left(\mathrm{P}_{f}, f\right)$ with $d\left(x_{2}, x\right)<\varepsilon / 10$. Hence, by the choice of $y$ (the $f$-orbit of $y$ is $\varepsilon_{1}$-dense in $\mathrm{H}\left(\mathrm{P}_{f}, f\right)$ and is equal to the $f_{1}$-orbit), there is some $i$ such that $f^{i}(y)=f_{1}^{i}(y)=y_{i} \in \mathrm{B}\left(x_{2}, \varepsilon_{1}+\varepsilon / 10\right)$. Therefore,

$$
\mathrm{B}\left(y_{i}, \varepsilon_{1}\right) \subset \mathrm{B}\left(x_{2}, 2 \varepsilon_{1}+\varepsilon / 10\right) \subset \mathrm{B}\left(x_{2}, \varepsilon\right) \text {. }
$$

Finally, by condition (d3) above, there is $i$ such that

$$
f_{2}^{i}\left(\mathrm{D}_{0}\right) \subset \mathrm{B}\left(y_{i}, \varepsilon_{1}\right) \subset \mathrm{B}\left(x_{2}, \varepsilon\right) .
$$

In the same way, the property $\left(\mathscr{P}^{\prime}\right)$ follows from the lower semi continuity of the homoclinic class $\mathrm{H}\left(\mathrm{P}_{g}, g\right)$ and from property (d3).

Since the previous argument holds for every sufficiently small $\varepsilon_{0}>0$, the proof of the density of $\mathscr{P}\left(\mathscr{O}, \varepsilon, \mathscr{W}_{0}\right)$ in $\mathscr{W}_{0}$ is complete. This also ends the proof of the lemma.

Consider now a countable basis $\mathscr{O}_{n}$ of the topology of $\operatorname{Diff}_{\text {int }}^{+}\left(\mathrm{D}^{3}\right)$ and a sequence $\varepsilon_{n}>0$ with $\varepsilon_{n} \rightarrow 0$ as $n \rightarrow+\infty$. Define the set

$$
\mathscr{U}_{0}=\bigcap_{i, j \in \mathbf{N}}\left(\mathscr{P}\left(\mathscr{O}_{i}, \varepsilon_{j}, \mathscr{W}_{0}\right) \cap \mathscr{P}^{-}\left(\mathscr{O}_{i}, \varepsilon_{j}, \mathscr{W}_{0}\right)\right) .
$$

By Lemma 3.10, the set $\mathscr{U}_{0}$ is a countable intersection of dense open subsets of $\mathscr{W}_{0}$, thus it is a residual subset of $\mathscr{W}_{0}$.

The first step of the proof of Theorem B is to see that it holds for a fixed wild homoclinic class $\mathrm{H}\left(\mathrm{P}_{f}, f\right) \in \mathrm{W}_{0}(f)$. This is a consequence of the next lemma:

Lemma 3.12. - Every $f \in \mathscr{U}_{0}$ has universal dynamics at $\mathrm{H}\left(\mathrm{P}_{f}, f\right)$ (recall Definition 1.3).

Proof. - Given $f \in \mathscr{U}_{0}$ denote by $\mathrm{D}_{i, j}^{+}$and $m_{i, j}^{+}$(resp. $\mathrm{D}_{i, j}^{-}$and $m_{i, j}^{-}$) the disk and the natural number (period of the disk) associated to $f$ by the condition $f \in \mathscr{P}\left(\mathscr{O}_{i}, \varepsilon_{j}, \mathscr{W}_{0}\right)$ (resp. $\left.f \in \mathscr{P}^{-}\left(\mathscr{O}_{i}, \varepsilon_{j}, \mathscr{W}_{0}\right)\right)$, and so verifying the conditions $\left(\mathscr{P}_{1}\right)-$ $(\mathscr{P} 5)$.

Claim 1. - There are two sequences $j(i)$ and $k(i)$ converging to $+\infty$ as $i \rightarrow+\infty$ and satisfying the following property: 
- given any integer $i \in \mathbf{N}$ denote by $\delta_{2 i}$ and $\delta_{2 i+1}$ the disks $\mathrm{D}_{i, j(i)}^{+}$and $\mathrm{D}_{i, k(i)}^{-}$, respectively, and consider the unions $\Delta_{2 i}=\bigcup_{n=0}^{+\infty} f^{n}\left(\delta_{2 i}\right)$ and $\Delta_{2 i+1}=$ $\bigcup_{n=0}^{+\infty} f^{-n}\left(\delta_{2 i+1}\right)$. Then the sets $\Delta_{\ell}, \ell \in \mathbf{N}$, are pairwise disjoint.

Before proving the claim, let us conclude the proof of Lemma 3.12. Consider any open set $\mathscr{O}$ of $\operatorname{Diff}_{\text {int }}^{+}\left(\mathrm{D}^{3}\right)$ and any point $x \in \mathrm{H}\left(\mathrm{P}_{f}, f\right)$. As the set $\mathscr{O}_{i}$ are a basis of the topology of $\operatorname{Diff}_{\text {int }}^{+}\left(\mathrm{D}^{3}\right)$, there is an infinite sequence $i_{n} \rightarrow+\infty$ such that $\mathscr{O}_{i_{n}} \subset \mathscr{O}$. Property $(\mathscr{P} 3)$ implies that, for any integer $i$, the disk $\delta_{2 i}$ has a positive iterate $\mathrm{D}_{2 i}$ contained in the ball $\mathrm{B}\left(x, \varepsilon_{j(i)}\right)$ and the disk $\delta_{2 i+1}$ has a negative iterate $\mathrm{D}_{2 i+1}$ contained in the ball $\mathrm{B}\left(x, \varepsilon_{j(i)}\right)$. Now one easily verifies that the sequence $\mathrm{D}_{k}, k \in \mathbf{N}$, verifies all the conditions $\left(\mathrm{U}_{1}\right)-\left(\mathbf{U}_{4}\right)$ in the definition of the universal dynamics, concluding the proof of the lemma.

We now prove the claim. By Remark 3.11, the homoclinic class $\mathrm{H}\left(\mathrm{P}_{f}, f\right)$ and the forward $f$-orbit of $\mathrm{D}_{i, j}^{+}$are compact disjoint sets, thus there is $\mu_{i, j}^{+}>0$ such that the distance between $\mathrm{H}\left(\mathrm{P}_{f}, f\right)$ and $\bigcup_{k \geq 0} f^{k}\left(\mathrm{D}_{i, j}^{+}\right)$is greater than $2 \mu_{i, j}^{+}$. We argue analogously with $\mathrm{H}\left(\mathrm{P}_{f}, f\right)$ and the backward $f$-orbit of $\mathrm{D}_{i, j}^{-}$, obtaining a positive lower bound $2 \mu_{i, j}^{-}$for the distance between $\mathrm{H}\left(\mathrm{P}_{f}, f\right)$ and $\bigcup_{k \leq 0} f^{k}\left(\mathrm{D}_{i, j}^{-}\right)$.

We build the sequences $j(i)$ and $k(i)$ inductively. Assume that $j(0), k(0), \ldots$, $j(i), k(i)$ are defined, we define $j(i+1)$ and $k(i+1)$ as follows: we choose $j(i+1)$ such that

$$
\varepsilon_{j(i+1)}<\inf \left\{\mu_{\ell, j(\ell)}^{+}, \mu_{\ell, k(\ell)}^{-}, \ell=0, \ldots, i\right\} .
$$

Property $\left(\mathscr{P} 3^{\prime}\right)$ asserts that the union of the positive iterates of the disk $\mathrm{D}_{i, j(i+1)}^{+}$is contained in the $\varepsilon_{j(i+1)}$ neighborhood of $\mathrm{H}\left(\mathrm{P}_{f}, f\right)$ so that $\Delta_{2(i+1)}$ is disjoint from the $\Delta_{\ell}, \ell \leq 2 i+1$. In the same way, we choose $k(i+1)$ such that

$$
\varepsilon_{k(i+1)}<\inf \left\{\mu_{i+1, j(i+1)}^{+}, \inf \left\{\mu_{\ell, j(\ell)}^{+}, \mu_{\ell, k(\ell)}^{-}, \ell=0, \ldots, i\right\}\right\} .
$$

This concludes the proof of the claim.

To end the proof of Theorem $\mathrm{B}$ we need to get the universal property for all the wild homoclinic classes in $\mathrm{W}_{0}(f)\left(f\right.$ in a residual subset of $\mathscr{W}_{0}(\mathrm{M})$ ). For that consider a countable basis $\mathscr{G}_{n}$ of the set of wild diffeomorphisms of $\mathscr{W}_{0}(\mathrm{M})$ and, for each $n$, consider all the pairs $\left(\mathscr{G}_{n}, \mathrm{P}: \mathscr{G}_{n} \rightarrow \mathrm{M}\right)$ where $\mathrm{P}$ is a continuous map associating to each $f$ a hyperbolic periodic point $\mathrm{P}(f)$ of $f$ with a wild homoclinic class in $\mathrm{W}_{0}(f)$. Observe that this set of pairs is countable. Thus we can index them in the form $\mathscr{Y}_{i}=\left(\mathscr{G}_{n(i)}, \mathrm{P}_{i}\right)$.

By Lemma 3.12 , there is a residual subset $\mathscr{Z}_{i}$ of $\mathscr{G}_{n(i)}$ such that every $f \in \mathscr{Z}_{i}$ has universal dynamics at $\mathrm{H}\left(\mathrm{P}_{i}(f), f\right)$.

Consider now $\mathscr{V}_{i}=\mathscr{Z}_{i} \cup\left(\mathscr{W}_{0}(\mathrm{M}) \backslash \mathscr{G}_{n(i)}\right)$. This set is residual in $\mathscr{W}_{0}(\mathrm{M})$. Then $\mathscr{V}=\bigcap_{i \in \mathbf{N}} \mathscr{V}_{i}$ is residual in $\mathscr{W}_{0}(\mathrm{M})$ and any $f \in \mathscr{V}$ has universal dynamics at each wild homoclinic class of $\mathrm{W}_{0}(f)$. This completes the proof of Theorem $\mathrm{B}$. 


\section{Aperiodic maximal transitive Cantor sets}

In this section we prove Theorem A.

\subsection{Notations}

By definition, every $f \in \mathscr{W}_{0}(\mathrm{M})$ has a saddle $\mathrm{P}_{f}$ such that $\mathrm{H}\left(\mathrm{P}_{f}, f\right)$ is a wild homoclinic class in $\mathrm{W}_{0}(f)$. Moreover, for every $f \in \mathscr{W}_{0}(\mathrm{M})$ there is a neighborhood where the points $\mathrm{P}_{g}$ depend continuously on $g$. Using the metrizability of $\operatorname{Diff}^{1}(\mathrm{M})$, one gets a dense open subset $\mathscr{W}_{1}(\mathrm{M}) \subset \mathscr{W}_{0}(\mathrm{M})$ and a continuous function $f \mapsto$ $\mathrm{P}_{0}(f)$ defined on $\mathscr{W}_{1}(\mathrm{M})$ such that $\mathrm{H}\left(\mathrm{P}_{0}(f), f\right) \in \mathrm{W}_{0}(f)$ for all $f \in \mathscr{W}_{1}(\mathrm{M})$.

We denote by $\mathscr{W}_{0}\left(\mathrm{D}^{3}\right) \subset \operatorname{Diff}_{\text {int }}^{+}\left(\mathrm{D}^{3}\right)$ the subset of diffeomorphisms $f$ having a wild homoclinic class in $\mathrm{W}_{0}(f)$ and, as above, consider a dense open subset $\mathscr{W}_{1}\left(\mathrm{D}^{3}\right)$ of $\mathscr{W}_{0}\left(\mathrm{D}^{3}\right)$ of diffeomorphisms with a point $\mathrm{P}(f)$ depending continuously on $f \in \mathscr{W}_{1}\left(\mathrm{D}^{3}\right)$ and having a wild homoclinic class in $\mathrm{W}_{0}(f)$.

Given $\varepsilon>0$, consider now the sets $\mathscr{P}(\mathrm{M}, \varepsilon)=\mathscr{P}\left(\mathscr{W}_{1}\left(\mathrm{D}^{3}\right), \varepsilon, \mathscr{W}_{1}(\mathrm{M})\right)$ defined as in Section 3.3 and associated to the open sets $\mathscr{O}=\mathscr{W}_{1}\left(\mathrm{D}^{3}\right)$ and $\mathscr{W}_{0}=$ $\mathscr{W}_{1}(\mathrm{M})$. By Lemma 3.10, the set $\mathscr{P}(\mathrm{M}, \varepsilon)$ is open and dense in $\mathscr{W}_{1}(\mathrm{M})$ for every $\varepsilon>0$.

We observed in Remark 3.9 that the disks $\mathrm{D}_{1}(f)$ and the natural numbers $k_{1}(f)$ verifying $(\mathscr{P} 1)-(\mathscr{P} 5)$, as well as the identification of $\mathrm{D}_{1}(f)$ with $\mathrm{D}^{3}$ conjugating $f^{k_{1}(f)}$ to some $g_{f} \in \mathscr{W}_{1}\left(\mathrm{D}^{3}\right)$, can be chosen varying locally continuously. Moreover, as the restriction of $f^{k_{1}(f)}$ to $\mathrm{D}_{1}(f)$ is conjugate to some $g_{f} \in \mathscr{W}_{1}\left(\mathrm{D}^{3}\right)$ (up to the identification $\left.\mathrm{D}_{1}(f)=\mathrm{D}^{3}\right), f$ has a periodic point $\mathrm{P}_{1}(f)$ corresponding to $\mathrm{P}\left(g_{f}\right)$, depending continuously on $f$ and having a wild homoclinic class in $\mathrm{W}_{0}(f)$. So there is a dense open subset $\mathscr{Q}_{1}(\mathrm{M}, \varepsilon) \subset \mathscr{P}(\mathrm{M}, \varepsilon)$ and a continuous function $\Psi_{1}$ defined on $\mathscr{Q}_{1}(\mathrm{M}, \varepsilon)$ by

$$
f \mapsto \Psi_{1}(f)=\left(\mathrm{D}_{1}(f), k_{1}(f), \mathrm{P}_{1}(f)\right),
$$

where

- $\mathrm{D}_{1}(f)$ and $k_{1}=k_{1}(f)>1$ are the disk and the natural number given by the definition of $\mathscr{P}(\mathrm{M}, \varepsilon)$, satisfying $(\mathscr{P} 1)-(\mathscr{P} 5)$, and such that the restriction of $f^{k_{1}}$ to the disk $\mathrm{D}_{1}(f)$ is differentially conjugate to some $g \in \mathscr{W}\left(\mathrm{D}^{3}\right)$, recall condition $(\mathscr{P} 5)$. Observe that, by $(\mathscr{P} 4)$, the diameters of the disks $f^{i}\left(\mathrm{D}_{1}(f)\right), i \in\left\{-k_{1}(f), \ldots, k_{1}(f)\right\}$, are upper bounded by $2 \varepsilon$.

- $\mathrm{P}_{1}(f) \in \mathrm{D}_{1}(f)$ is a point with a wild homoclinic class in $\mathrm{W}_{0}(f)$.

We define analogously the set $\mathscr{Q}_{1}^{+}\left(\mathrm{D}^{3}, \varepsilon\right) \subset \mathscr{W}_{0}\left(\mathrm{D}^{3}\right)$ and the maps $\mathrm{D}_{1}(f)$, $k_{1}(f)$ and $\mathrm{P}_{1}(f)$. We define $\mathscr{Q}_{1}^{-}\left(\mathrm{D}^{3}, \varepsilon\right)$ as the set of diffeomorphisms $f$ such that $f^{-1}$ belongs to $\mathscr{Q}_{1}^{+}\left(\mathrm{D}^{3}, \varepsilon\right)$. 


\subsection{The inductive process}

For starting our construction we fix a sequence $\varepsilon_{i}>0, i \in \mathbf{N}^{*}$, converging to 0 as $i \rightarrow \infty$.

Let $\mathscr{Q}_{1}=\mathscr{Q}_{1}\left(\mathrm{M}, \varepsilon_{0}\right)$. Notice that for any $f \in \mathscr{Q}_{1}$ there is $\varepsilon_{1}(f)$ such that, for any disk $\Delta^{\prime}$ of diameter less than $2 \varepsilon_{1}(f)$ in $\mathrm{D}^{3}$, one has the following property: denote by $\Delta \subset \mathrm{D}_{1}(f)$ the disk corresponding to $\Delta^{\prime}$ via the identification, then the iterates $f^{i}(\Delta), i \in\left\{-k_{1}(f), \ldots, k_{1}(f)\right\}$, have diameters bounded by $\varepsilon_{1}$.

As the identification of $\mathrm{D}_{1}(f)$ with $\mathrm{D}^{3}$ depends continuously on $f$, the number $\varepsilon_{1}(f)$ can be chosen locally independent of $f$. So there is a dense open subset $\mathscr{R}_{1}$ of $\mathscr{Q}_{1}$ where $\varepsilon_{1}(f)$ is locally constant.

Define the set $\mathscr{Q}_{2}$ as the subset of $\mathscr{R}_{1}$ of diffeomorphisms $f$ such that the restriction of $f^{k_{1}(f)}$ to $\mathrm{D}_{1}(f)$ is identified to some element $g_{f} \in \mathscr{Q}_{1}^{-}\left(\mathrm{D}^{3}, \varepsilon_{1}(f)\right)$. Remark that, by Lemma 3.10 , the set $\mathscr{Q}_{2}$ is open and dense in $\mathscr{R}_{1}$. Consider $f \in \mathscr{Q}_{2}$ and let

- $\mathrm{D}_{2}(f)$ be the disk identified with $\mathrm{D}_{1}\left(g_{f}\right)$,

- $k_{2}(f)=k_{1}(f) \cdot k_{1}\left(g_{f}\right) \geq 2 \cdot k_{1}(f)$,

- $\mathrm{P}_{2}(f) \in \mathrm{D}_{2}(f)$ be the point identified with $\mathrm{P}_{1}\left(g_{f}\right)$ (with a wild homoclinic class in $\left.\mathrm{W}_{0}\left(g_{f}\right)\right)$.

Lemma 4.1. - The diameter of $f^{i}\left(\mathrm{D}_{2}(f)\right)$ is upper bounded by $\varepsilon_{1}$ for every $i \in$ $\left\{-k_{2}(f), \ldots, k_{2}(f)\right\}$ and every $f \in \mathscr{Q}_{2}$.

Proof. - Write $i=j+l \cdot k_{1}(f)$, with $j \in\left\{-k_{1}(f), \ldots, k_{1}(f)\right\}$ and $l \in\left\{-k_{2}\left(g_{f}\right)\right.$, $\left.\ldots, k_{2}\left(g_{f}\right)\right\}$. The disk $g^{l}\left(\mathrm{D}_{1}\left(g_{f}\right)\right)$ has diameter less than $2 \varepsilon_{1}(f)$ (recall $\left.(\mathscr{P} 3)\right)$, and the corresponding disk (via the identification) in $\mathrm{D}_{1}(f)$ is $f^{l \cdot k_{1}\left(g_{f}\right)}\left(\mathrm{D}_{2}(f)\right)$. The choice of $\varepsilon_{1}(f)$ now implies that $f^{j}\left(f^{l \cdot k_{1}\left(g_{f}\right)}\left(\mathrm{D}_{2}(f)\right)\right)$ has diameter less than $\varepsilon_{1}$, finishing the proof of the lemma.

We now construct inductively nested sequences $\mathscr{Q}_{n}$ of subsets of $\mathscr{W}_{0}(\mathrm{M})$ (with continuous functions $\mathrm{D}_{n}(f), k_{n}(f)$ and $\left.\mathrm{P}_{n}(f)\right)$ and $\mathscr{R}_{n} \subset \mathscr{Q}_{n}$ (open and dense in $\mathscr{Q}_{n}$ and with a locally constant function $\left.\varepsilon_{n}(f)\right)$ in the following way:

- $\mathscr{Q}_{n+1}$ is the subset of $\mathscr{R}_{n}$ of diffeomorphisms $f$ such that the restriction of $f^{k_{n}(f)}$ to $\mathrm{D}_{n}(f)$ is identified (conjugate) with some $g_{f} \in \mathscr{Q}_{1}^{ \pm}\left(\mathrm{D}^{3}, \varepsilon_{n}(f)\right)$, where \pm is the sign of $(-1)^{n}$.

- Thereafter we construct $\mathscr{R}_{n+1} \subset \mathscr{Q}_{n+1}$, open and dense in $\mathscr{Q}_{n+1}$, with a locally constant function $\varepsilon_{n+1}$ such that for every disk $\Delta^{\prime}$ of diameter less than $\varepsilon_{n+1}(f)$ in $\mathrm{D}^{3}$ the corresponding disk $\Delta \subset \mathrm{D}_{n+1}(f)$ via the identification is such that the iterates $f^{i}(\Delta), i \in\left\{-k_{n+1}(f), \ldots, k_{n+1}(f)\right\}$, have diameters bounded by $\varepsilon_{n+1}$. 
By construction, the sets and the functions above satisfy the following properties:

1. $\mathscr{Q}_{n}$ is open and dense in $\mathscr{Q}_{n-1}$, so it is also open and dense in $\mathscr{W}_{0}(\mathrm{M})$,

2. $k_{n+1}(f)=m_{n} \cdot k_{n}(f)$ for some $m_{n} \geq 2$,

3. the diameters of $f^{-k_{n}(f)}\left(\mathrm{D}_{n}(f)\right), \ldots, f^{k_{n}(f)}\left(\mathrm{D}_{n}(f)\right)$ are upper bounded by $\varepsilon_{n-1}$, in particular, these diameters uniformly go to 0 as $n \rightarrow \infty$,

4. if $n$ is even then the disks $\mathrm{D}_{n}(f), f\left(\mathrm{D}_{n}(f)\right), \ldots, f^{k_{n}(f)-1}\left(\mathrm{D}_{n}(f)\right)$ are pairwise disjoint and $f^{k_{n}(f)}\left(\mathrm{D}_{n}(f)\right) \subset \operatorname{int}\left(\mathrm{D}_{n}(f)\right)$,

5. if $n$ is odd then the disks $\mathrm{D}_{n}(f), f^{-1}\left(\mathrm{D}_{n}(f)\right), \ldots, f^{-k_{n}(f)+1}\left(\mathrm{D}_{n}(f)\right)$ are pairwise disjoint and $f^{-k_{n}(f)}\left(\mathrm{D}_{n}(f)\right) \subset \operatorname{int}\left(\mathrm{D}_{n}(f)\right)$,

6. for every even (resp. odd) number $n$ the positive (resp. negative) $f$-orbit of $\mathrm{D}_{n}(f)$ is contained in the negative (resp. positive) orbit of $\mathrm{D}_{n-1}(f)$.

Consider now the residual subset $\mathscr{R}=\bigcap_{n \geq 1} \mathscr{Q}_{n}$ of $\mathscr{W}_{0}(\mathrm{M})$. For each $f \in \mathscr{R}$ define the set

$$
\Phi(f)=\bigcap_{n \in \mathbf{N}} \bigcup_{0}^{k_{2 n}(f)} f^{i}\left(\mathrm{D}_{2 n}(f)\right) .
$$

Theorem A follows from the next proposition:

Proposition 4.2. - Consider $f$ in the residual subset $\mathscr{R}$ of $\mathscr{W}_{0}(\mathrm{M})$. Then the following holds:

- $\Phi(f)$ is an $f$-invariant Cantor set.

- The forward orbit of any point $x \in \Phi(f)$ is dense in $\Phi(f)$, that is, $f$ is minimal on $\Phi(f)$ (in particular, $\Phi(f)$ is transitive and aperiodic).

- $\Phi(f)$ is a maximal transitive set and it is saturated.

- $\Phi(f)$ is Lyapunov stable for $f$ and $f^{-1}$.

- $\Phi(f)$ is uniquely ergodic.

Proof. - The majoration by $\varepsilon_{n-1}$ of the diameters of the disks $f^{i}\left(\mathrm{D}_{n}(f)\right)$, $i=-k_{n}, \ldots, k_{n}(f)$, implies that $\Phi(f)$ is totally disconnected. The fact that $\mathrm{D}_{n}(f)$ contains $m_{n}>1$ disks of the orbit of $\mathrm{D}_{n+1}$ implies that the set $\Phi(f)$ has no isolated points. Finally, it is compact as intersection of compact sets. Therefore the set $\Phi(f)$ is a Cantor set.

Consider the set $\bigcup_{0}^{k_{2 n}(f)-1} f^{i}\left(\mathrm{D}_{2 n}(f)\right)$ (resp. $\left.\bigcup_{0}^{k_{2 n+1}(f)-1} f^{-i}\left(\mathrm{D}_{2 n+1}(f)\right)\right)$. Observe that, for each $n$, this set is a forward (resp. backward) invariant $\left(\varepsilon_{2 n-1}\right)$-neighborhood (resp. $\left(\varepsilon_{2 n}\right)$-neighborhood) of $\Phi(f)$, thus the set $\Phi(f)$ is $f$-invariant and Lyapunov stable for $f$ and $f^{-1}$.

Observe that, by construction, the set $\Phi(f)$ does not contain periodic points: each orbit of $\Phi(f)$ meets any disk $\mathrm{D}_{n}(f)$, whose $k_{n}(f)-1$ first iterates are disjoint, thus its period is at least $k_{n}(f)$ for all $n$. As $k_{n}(f) \rightarrow+\infty$, there is no periodic 
points in $\Phi(f)$. Moreover, by construction, each orbit of $\Phi(f)$ meets each component of $\bigcup_{0}^{k_{2 n}(f)-1} f^{i}\left(\mathrm{D}_{2 n}(f)\right)$, implying that each orbit of $\Phi(f)$ is $\left(\varepsilon_{2 n-1}\right)$-dense for every $n$. Since $\varepsilon_{n} \rightarrow 0$, this implies the density of any forward orbit the proof for backward orbits is analogous). So the restriction of $f$ to $\Phi(f)$ is minimal and aperiodic.

The Lyapunov stability of $\Phi(f)$ implies that $\Phi(f)$ is maximal and saturated.

By construction, the time average of any orbit of $\Phi(f)$ in a disk $f^{i}\left(\mathrm{D}_{n}(f)\right)$ is equal to $\frac{1}{k_{n}(f)}$. One deduces from this fact that given any continuous function $\theta: \Phi(f) \rightarrow \mathbf{R}$, its Birkhoff sums along two orbits converge to the same number. Thus $\Phi(f)$ supports a unique invariant measure, which is necessarily uniquely ergodic. The proof of the proposition is now complete.

\section{Proof of Corollary G}

To prove Corollary C, observe that the property of having sinks, sources, non-trivial hyperbolic or partially hyperbolic robustly transitive attractors or repellors, homoclinic classes containing persistently points of different indices, and wild homoclinic classes in $\mathrm{W}_{0}(f)$ are robust properties, and that to exhibit an aperiodic maximal transitive set is a locally generic property, recall Theorem A. For the independent saddles, we can guarantee their robust existence using filtrations.

The unique difficulty to prove the corollary is to verify that these phenomena may appear in $\mathrm{D}^{3}$ : that is clear for sinks, sources and independent saddles. In $\left[\mathrm{BD}_{2}\right]$ it is explained how to get a wild homoclinic class in $\mathrm{W}_{0}(f)$, see also the appendix (Section 6). For hyperbolic attractors of dimension 1 one can consider, for example, the product of a Plykin attractor in $\mathrm{D}^{2}$ by a transverse contraction, or the Smale's solenoid (as far as we know, it is not possible to have 2-dimensional hyperbolic attractors in $\mathrm{D}^{3}$ ). For partially hyperbolic attractors, we consider the map defined on the solid torus $\mathrm{D}^{2} \times \mathrm{S}^{1}$ obtained as the product of a Plykin attractor in $\mathrm{D}^{2}$ by the identity map in $\mathrm{S}^{1}$. In $\left[\mathrm{BD}_{1}\right]$ it is proved that this map can be perturbed to get a robustly transitive partially hyperbolic (nonhyperbolic) attractor.

By Remark 1.2, the existence of wild homoclinic class in $\mathrm{D}^{3}$ implies the local genericity of diffeomorphisms of $\mathrm{D}^{3}$ having aperiodic maximal transitive sets.

The heuristic principle now implies that these phenomena appear generically infinitely many times in $\mathscr{W}_{0}(\mathrm{M})$.

Finally, observe that all the aperiodic maximal transitive sets in our construction are contained in the closure of the union of infinitely many wild homoclinic classes, and that the universal dynamics at the wild homoclinic classes allow us to get (generically) the other inclusion. 


\section{Appendix}

In this section we sketch the construction of wild homoclinic classes in $\mathrm{W}_{0}(f)$ whose existence was claimed in Section 1.1. This construction is a minor modification of the one in $\left[\mathrm{BD}_{2}\right.$, Section 3.2] involving blenders. Let us sketch this construction in the simplest situation.

In rough terms, a blender of a diffeomorphism $f$ is a cube $\Gamma$ endowed with $f$-invariant cone fields $\mathrm{C}^{s}, \mathrm{C}^{u}$ and $\mathrm{C}^{u u}, \mathrm{C}^{u u} \subset \mathrm{C}^{u}$, such that for every point $x \in \Gamma$ with $f(x) \in \Gamma$ the derivative of $f$ uniformly contracts the vectors in $\mathrm{C}^{s}$ and uniformly expands the vectors in $\mathrm{C}^{u}$. We assume that the cube $\Gamma$ contains a hyperbolic fixed saddle $Q$ of index 1 whose local stable manifold transversely meets the two sides of $\Gamma$ parallel to the unstable cone field. Using the cone field $\mathrm{C}^{m u}$, one defines vertical segments through $\Gamma$ as curves joining the top and the bottom faces of the cube and whose tangent vectors are in $\mathrm{C}^{m u}$. Using the manifold $\mathrm{W}_{\text {loc }}^{s}(\mathrm{Q})$, one speaks of vertical segments at the right and at the left of $\mathrm{W}_{l o c}^{s}(\mathrm{Q})$. Using this terminology, we say that $(\Gamma, f)$ is a blender associated to $Q$ if the closure of the stable manifold of $Q$ intersects every vertical segment through $\Gamma$ at the (say) right of $\mathrm{Q}$.

Suppose now that $(\Gamma, f)$ is a blender associated to a saddle $\mathrm{Q}$ of index 1 as above and that there is a hyperbolic fixed saddle $\mathrm{P}$ of index 2 whose onedimensional unstable manifold contains a vertical segment through $\Gamma$ at the right of $\mathrm{W}_{l o c}^{s}(\mathrm{Q})$. Then we say that $\mathrm{P}$ activates the blender $(\Gamma, f)$. In such a case, as a consequence of the $\lambda$-lemma, the whole stable manifold of $\mathrm{P}$ is contained in the closure of $\mathrm{W}^{s}(\mathrm{Q})$. Moreover, if $\mathrm{W}^{s}(\mathrm{P})$ and $\mathrm{W}^{u}(\mathrm{Q})$ have a nonempty transverse intersection, the whole homoclinic class of $\mathrm{P}$ is contained in $\mathrm{Q}$.

We observe that to have a blender is a $\mathrm{C}^{1}$-open property, this mainly follows since the existence of cone fields as above is $\mathrm{C}^{1}$-persistent and compact parts of invariant manifolds depend continuously on the diffeomorphism.

A simple way to get blenders is to consider a pair of saddles $\mathrm{P}$ and $\mathrm{Q}$ as above related by a heterodimensional cycle which is far from tangencies. Recall that a diffeomorphism $f$ has heterodimensional cycle associated to $\mathrm{P}$ and $\mathrm{Q}$ if $\mathrm{W}^{u}(\mathrm{P})$ and $\mathrm{W}^{s}(\mathrm{Q})$ meet quasi-transversely along an orbit and $\mathrm{W}^{s}(\mathrm{P})$ and $\mathrm{W}^{u}(\mathrm{Q})$ have a non-empty transverse intersection. This cycle is far from tangencies if every diffeomorphism $\mathrm{C}^{1}$-close to $f$ has no homoclinic tangencies associated to $\mathrm{P}$ or $\mathrm{Q}$. For this result see [DR]. In fact, in [DR] it is proved that, if $f$ has a cycle far from tangencies associated to $\mathrm{P}$ and $\mathrm{Q}$ there is an open set $\mathscr{B}$ of diffeomorphisms, $f \in \overline{\mathscr{B}}$, such that for every $g \in \mathscr{B}$ there are blenders $(\Gamma, f)$ associated to Q and activated by $\mathrm{P}$, and $\left(\Gamma, f^{-1}\right)$ associated to $\mathrm{P}$ and activated by $\mathrm{Q}$. Using the first blender one has $\mathrm{H}\left(\mathrm{P}_{g}, g\right) \subset \mathrm{H}\left(\mathrm{Q}_{g}, g\right)$, and the second one gives $\mathrm{H}\left(\mathrm{Q}_{g}, g\right) \subset$ $\mathrm{H}\left(\mathrm{P}_{g}, g\right)$ for all $g \in \mathscr{B}$. 
The previous construction is semi-local and it only involves the separatrices of $\mathrm{W}^{s}(\mathrm{Q})$ and of $\mathrm{W}^{u}(\mathrm{P})$ involved in the cycle. Thus it can be carried out satisfying also the following.

- The saddle $\mathrm{P}$ is homoclinically related to a saddle $\mathrm{P}^{\prime}$ having a pair of contracting non-real eigenvalues and such that the Jacobian of $f$ at $\mathrm{P}^{\prime}$ is bigger than one (for that it is enough to take $\mathrm{P}^{\prime}$ having a big expansion in the unstable direction).

- The saddle $Q$ is homoclinically related to a saddle $Q^{\prime}$ having a pair of expanding non-real eigenvalues and such that the Jacobian of $f$ at $\mathbf{Q}^{\prime}$ is less than one.

Since these conditions are $\mathrm{C}^{1}$-open, after shrinking $\mathscr{B}$ if necessary, we have that, for every $g \in \mathscr{B}$,

$$
\mathrm{H}\left(\mathrm{P}_{g}^{\prime}, g\right)=\mathrm{H}\left(\mathrm{P}_{g}, g\right)=\mathrm{H}\left(\mathrm{Q}_{g}, g\right)=\mathrm{H}\left(\mathrm{Q}_{g}^{\prime}, g\right) .
$$

Thus the homoclinic class $\mathrm{H}\left(\mathrm{P}_{g}, g\right)=\mathrm{H}\left(\mathrm{Q}_{g}, g\right)$ is wild and belongs to $\mathrm{W}_{0}(g)$ in the sense of Definition 1.1. This ends our sketch of the construction of wild homoclinic classes.

\section{REFERENCES}

[Ab] F. Abdenur, Generic robustness of a spectral decompositions, to appear in Ann. Scient. Éc. Norm. Sup..

[AS] R. Abraham and S. Smale, Nongenericity of $\Omega$-stability, Global Analysis I, Proc. Symp. Pure Math. A.M.S., 14 (1968), 5-8.

[Ar] M.-G. Arnaud, Creation de connexions en topologie $\mathrm{C}^{1}$, Ergod. Th. Dynam. Syst., 21 (2001), 339-381.

$\left[\mathrm{BD}_{1}\right]$ Ch. Bonatti and L. J. Díaz, Persistent nonhyperbolic transitive diffeomorphims, Ann. Math., 143 (1996), 357-396.

$\left[\mathrm{BD}_{2}\right]$ Ch. Bonatti and L. J. Díaz, Connexions hétéroclines et généricité d'une infinité de puits ou de sources, Ann. Scient. Éc. Norm. Sup., $4^{e}$ série, t32 (1999), 135-150.

[BDP] Ch. Bonatti, L. J. Díaz and E. R. Pujals, A $\mathscr{C}^{1}$-generic dichotomy for diffeomorphisms: Weak forms of hyperbolicicity or infinitely many sinks or sources, to appear in Annals of Math.

[BGLT] Ch. Bonatti, J.-M. Gambaudo, J.-M. Lion and Ch. Tresser, Wandering domains for infinitely renormalizable diffeomorphisms of the disk, Proc. Amer. Math. Soc., 122 (2) (1994), 1273-1278.

[BS] J. Buescu and I. Stewart, Liapunov stability and adding machines, Ergodic Th. Dynam. Syst., 15 (2) (1995), 271-290.

[CM] G. Carballo and C. Morales, Homoclinic classes and finitude of attractors for vector fields in $n$-manifolds, preprint (2001).

[GMP] G. Carballo, G. Morales and M. J. Pacífico, Homoclinic classes for $\mathscr{C}^{1}$-generic vector fields, to appear in Ergodic Th. Dynam. Syst.

[Ce] J. Cerf, Sur les difféomorphismes de la sphère de dimension trois $\left(\Gamma_{4}=0\right)$, Lecture Notes in Mathematics $\mathbf{5 3}$ (1968), pp. xii + 133, Springer-Verlag.

[DPU] L. J. Díaz, E. R. Pujals and R. Ures, Partial hyperbolicity and robust transitivity, Acta Math., 183 (1999), $1-43$.

[DR] L. J. Díaz and J. Rocha, Partially hyperbolic and transitive dynamics generated by heteroclinic cycles, Ergodic Th. Dyn. Syst., 25 (2001), 25-76.

[DS] L. J. Díaz and B. Santoro, Collision, explosion and collapse of homoclinic classes, preprint (2002).

[Fr] J. Franks, Necessary conditions for stability of diffeomorphisms, Trans. A.M.S., 158 (1971), 301-308. 
[Fu] H. Furstenberg, Strict ergodicity and transformations of the torus, Amer. F. of Math., 83 (1961), 573-601.

$[\mathrm{H}] \quad$ S. HAYAshi, Connecting invariant manifolds and the solution of the $\mathrm{C}^{1}$-stability and $\Omega$-stability conjectures for flows, Ann. of Math., 145 (1997), 81-137.

[Hu] M. Hurley, Attractors: persistence and density of their basins, Trans. Amer. Math. Soc., 269 (1982), $247-271$.

[Ma] R. Mañé, An ergodic closing lemma, Ann. of Math., 116 (1982), 503-540.

[Mi] J. W. Milnor, Topology from differential view point, Charlottesville, The University Press of Virginia, 1965.

$\left[\mathrm{N}_{1}\right] \quad$ S. Newhouse, Diffeomorphisms with infinitely many sinks, Topology, 13 (1974), 9-18.

$\left[\mathrm{N}_{2}\right] \quad$ S. Newhouse, The abundance of wild hyperbolic sets and nonsmooth stable sets for diffeomorphisms, Publ. Math. IHES, 50 (1979), 101-151.

[PT] J. Palis and F. TAkens, Hyperbolicity and sensitive chaotic dynamics at homoclinic bifurcations, Cambridge Studies in Advanced Mathematics, 35 (1993).

[PV] J. Palis and M. Viana, High dimension diffeomorphisms displaying infinitely many sinks, Ann. of Math., 140 (1994), 207-250.

[Pu] C. Pugh, The closing lemma, Amer. Four. of Math., 89 (1967), 956-1009.

[Si] R. C. Simon, A 3-dimensional Abraham-Smale example, Proc. A.M.S., 34 (2) (1972), 629-630.

[Sm] S. Smale, Differentiable dynamical systems, Bull. A.M.S., 73 (1967), 747-817.

[Tj] J. C. Tatjer, Three dimensional dissipative diffeomorphisms with homoclinic tangencies, Ergod. Th. Dynam. Syst., 21 (2001), 249-302.

C. B.

Laboratoire de Topologie, UMR 5584 du CNRS,

BP 47 870,

21078 Dijon Cedex,

France,

bonatti@u-bourgogne.fr

L. J. D.

Dep. Matemática PUC-Rio,

Marquês de S. Vicente 225,

22453-900 Rio de Janeiro RJ,

Brazil,

lodiaz@mat.puc-rio.br

Manuscrit reçu le 13 décembre 2000. 